\title{
Engineering of a nepetalactol-producing platform strain of Saccharomyces cerevisiae for the production of plant seco- iridoids
}

Alex Campbell

\author{
A Thesis \\ in \\ The Department \\ of
}

Biology
Presented in Partial Fulfillment of the Requirements
for the Degree of Master of Science (Biology) at Concordia University
Montreal, Quebec, Canada

September 2016

${ }^{\circledR}$ Alex Campbell 
CONCORDIA UNIVERSITY School of Graduate Studies

This is to certify that the thesis prepared

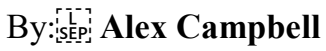

Entitled:i the production of plant seco-iridoids

and submitted in partial fulfillment of the requirements for the degree of : Masters of Biology

complies with the regulations of the University and meets the accepted standards with respect to originality and quality.

Signed by the final examining committee:

Dr. Grant Brown

Chair

Dr. Peter Pawelek

Examiner

Dr. Chris Brett

Examiner

Dr. Vincent Martin

Supervisor

\section{Dr. Patrick Gulick}

Chair of Department or Graduate Program Director

Dean of Faculty

Date $\_\underline{14 / 09 / 2016}$ 


\section{ACKNOWLEDGEMENTS}

First and foremost, I would like to thank my supervisor Vincent Martin for his guidance, encouragement, and trusting me not to blow up his lab. This experience has allowed me to develop my knowledge synthetic biology and I have greatly enjoyed working here over the years, so thanks Vince!

I would also like to thank the Martin lab for providing a friendly, supportive environment and generally always being ready to provide help and advice when needed. Particular individuals that stand out are James Scrivens, who helped me learn the initial techniques and Nick Gold. This work would not have been possible without Nick's patience and knowledge and I am grateful to him both for the technical skills he taught me as well as for being a great guy to work with.

I'd also like to thank Zach Wiltshire, Shoham Mookerjee and Marshall Timmermans. This thesis...well it probably would have been done a lot sooner without your antics, but I had infinitely more fun doing it.

Lastly, I'd like to thank my committee Dr. Peter Pawlelek and Dr. Chris Brett for their help and guidance throughout this project. 


\begin{abstract}
The monoterpene indole alkaloids (MIAs) are a valuable family of chemicals that include the anti-cancer drugs vinblastine and vincristine. These compounds are of global significance - appearing on the World Health Organization's list of model essential medicines - but remain exorbitantly priced due to low in planta levels. Chemical synthesis and genetic manipulation of MIA producing plants such as Catharanthus roseus have so far failed to find a solution to this problem. Synthetic biology holds a potential answer, by building the pathway into more tractable organisms such as Saccharomyces cerevisiae. Recent work has taken the first steps in this direction by producing small amounts of the intermediate strictosidine in yeast. In order to help improve on these titers, we aimed to optimize the early biosynthetic steps of the MIA pathway to the metabolite nepetalactol. We combined a number of strategies to create a base strain producing $11.4 \mathrm{mg} / \mathrm{L}$ of the precursor geraniol. We also show production of the critical intermediate 10-hydroxygeraniol and demonstrate nepetalactol production in vitro. We demonstrate that activity of the Iridiod synthase towards the intermediates geraniol and 10-hydroxygeraniol results in the synthesis of the non-productive intermediates citronellol and 10-hydroxycitronellol. As a means of improving metabolic flux in this system, we also experimented with the use of protein scaffolds to co-localize vulnerable pathway enzymes together. We demonstrate that protein scaffolds successfully bind their targets in vivo, and as a test case showed we could raise the production of citronellol up to $57.8 \%$. These discoveries have serious implications for the reconstruction of the MIA in heterologous organisms.
\end{abstract}




\section{Table of Contents}

List of tables

List of figures. Error! Bookmark not defined.

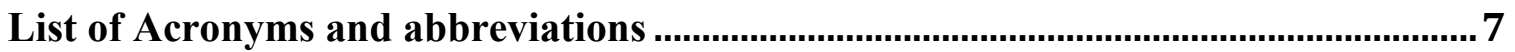

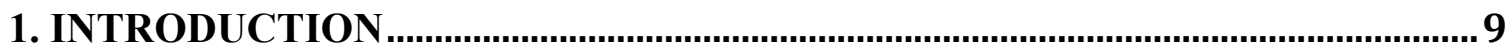

1.1 The Monoterpenoid Indole Alkaloids........................................................................

1.2 Production strategies and economics of the monoterpenoid indole alkaloids ............... 11

1.2.1 Chemical Synthesis of MIA's ...............................................................................................11

1.2.2 Metabolic Engineering of $C$. roseus ......................................................................................13

2.2.3 In vitro Cell Cultures for MIA Production...........................................................................14

2.2.4 Summary of Production Systems for the Monoterpene Indole Alkaloid's.......................14

2.3 MIA biosynthesis and pathway ....................................................................................15

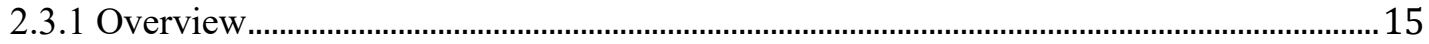

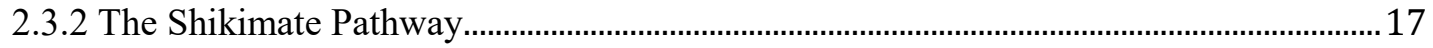

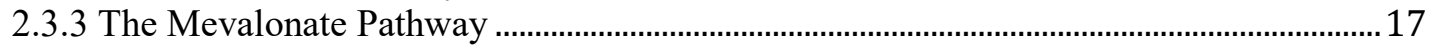

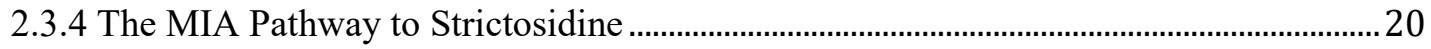

2.3.5 The MIA Pathway to Vinblastine/Vincristine..................................................................22

2.4 General Reconstruction Strategy ……......................................................................... 24

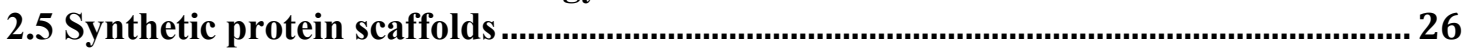

MATERIALS AND METHODS........................................................................... 27

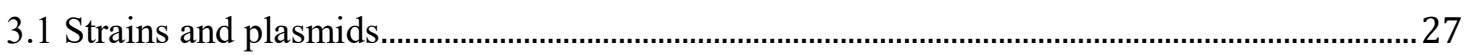

3.2 Integration of the $\operatorname{erg} 20^{\mathrm{K} 197 \mathrm{E}}$ mutation.................................................................................36

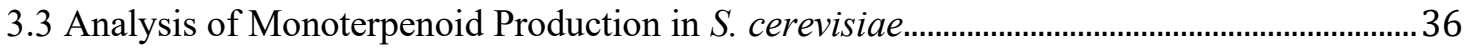

3.4 10-Hydroxygeraniol Oxidoreductase and Iridoid Synthase Assays............................................37

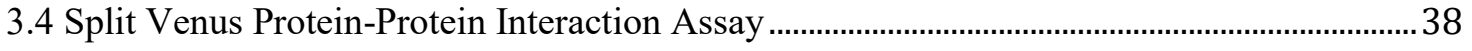

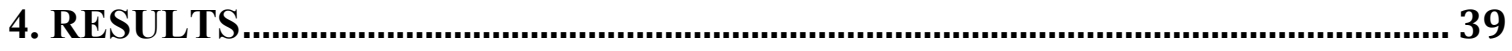

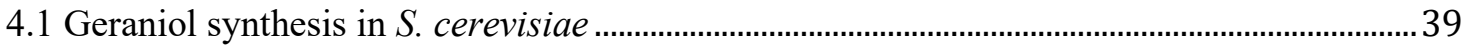

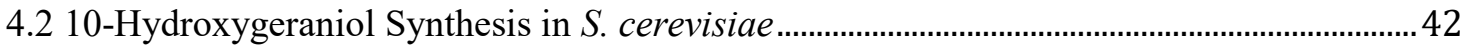

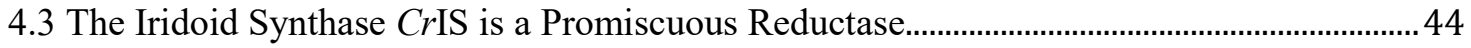

4.4 In vitro enzyme activity of $\mathrm{Cr} 10 \mathrm{HGO}-\mathrm{CrIS}$ enzyme pair.......................................................... 47

4.5 In vivo Characterization of Protein-Protein Binding Domains...................................................49

4.6 Enhanced Production of MIA Metabolites using Protein Scaffolds.......................................50

5. DISCUSSION

5.1 Improvement of Monoterpene Production ..............................................................................54

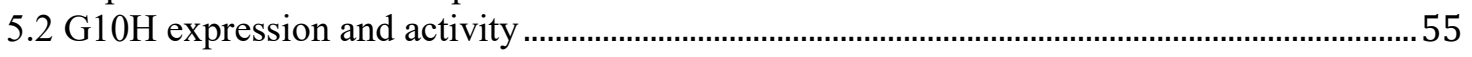

5.3 Impact of Iridiod synthase on Metabolite Distribution ..............................................................56

5.4 Enzyme Co-localization and the MIA Pathway ……………………………………………...57

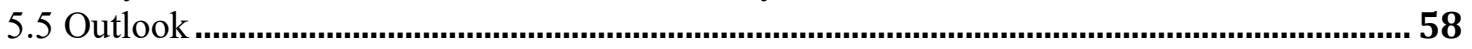

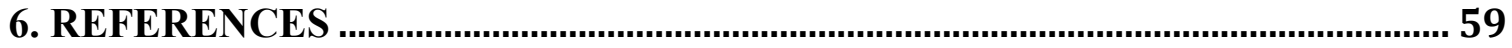




\section{List of tables}

Table 1: List of pharmaceutically active MIA's and their functions......................................10

Table 2: Strains and plasmids used in this work....................................29

Table 3: Oligonucleotides used in the work .......................................

\section{List of figures}

Figure 1: Chemical Structure of a) Vinblastine b) Vincristine...............................................12

Figure 2: Overview of the MIA Pathway............................................................................. 16

Figure 3: The mevalonate pathway from S. cerevisiae ........................................................ 19

Figure 4: The "early" MIA pathway from GPP to strictosidine. ………………………....2 21

Figure 5: The MIA pathway from strictosidine to Vinblastine/Vincristine. ................. 23

Figure 6: Metabolic engineering strategy to achieving nepetalactol synthesis in

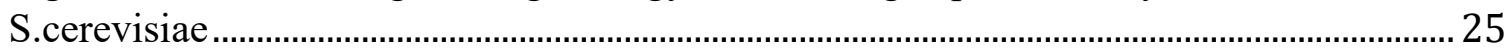

Figure 7:General structure of a synthetic protein scaffold:..................................................2

Figure 8: Split Venus protein-protein interaction assay................................................... 39

Figure 9: Specific production of the monoterpenoids and mevalonate by engineered

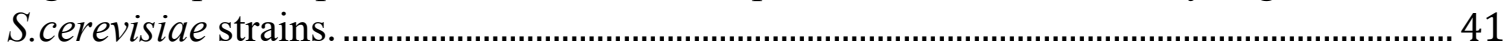

Figure 10: Production of monoterpenoids from geraniol-producing strains without ($\mathrm{CrG10H})$ and with $(+\mathrm{CrG10H})$ heterologous expression of the C.roseus geraniol 10-

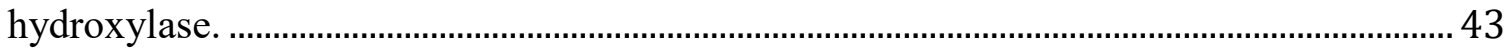

Figure 11: Evidence of promiscuity of CrIS. …………………………………………... 46

Figure 12: 10-hydroxygeraniol oxidase and irodidial synthase enzyme assays......... 48

Figure 13: Split Venus protein-protein interaction assay..................................................... 50

Figure 14: Depiction of citronellol based scaffolding experiments.....................51

Figure 15: Monoterpene production time course in the presence of different

scaffolds. 


\section{List of Acronyms and abbreviations}

10HGO: 10-hydroxygeraniol hydroxylase

Amp: Ampicillin

Anhydrovinblastine: AVB

CPR: Cytochrome p450 reductase

$\mathrm{CR}$ : Cathenamine reductase

C.Roseus: Catharanathus roseus

$\mathrm{CV}$ : C-terminal Venus fluorescent protein fragment

DNA: deoxyribonucleic acid

DMAPP Dimethylallyl pyrophosphate

E.coli: Escherichia coli

FPP: Farnesyl pyrophosphate

G10H: Geraniol 10 hydroxylase

GPP: Geranyl pyrophosphate

GES: Geraniol synthase

G10H: Geraniol-10 hydroxylase

10HGO: 10-hydroxygeraniol oxidase

HMG-CoA: 3-hydroxymethylglutaryl-CoA

HMGr: 3-hydroxymethylglutaryl-CoA reductase

IS: Iridoid synthase

LB: Lysogeny broth

MIA: Monoterpeniod indole alkaloids

MVP: Mevalonate pathway

$\mathrm{NV}$ : N-terminal Venus fluorescent protein fragment

OYE2/3: Old yellow enzyme 2/3

PEG: polyethylene glycol

PCR: Polymerase chain reaction

S.cerevisiae: Saccharomyces cerevisiae 
STR: Strictosidine synthase

SLS: Secologanin synthase

TDC: Tryptophan decarboxylase

VBL: Vinblastine

VCR: Vincristine

YPD: Yeast peptone dextrose

YNB: Yeast nitrogen base 


\section{INTRODUCTION}

\subsection{The Monoterpenoid Indole Alkaloids}

The monoterpene indole alkaloids (MIAs) are a large and chemically diverse class of naturally occurring plant metabolites. They are defined by a common chemical core, which consists of a terpene component containing the glycolsated Iridiod secologanin and an indole component comprising tryptamine (Figure 2). This natural scaffold gives rise to over 3000 different molecules that are produced by thousands of plant species [1]. Scientific interest in the MIA's is primarily due to their wide-ranging and potent pharmacological effects. Well known examples include the antimalarial quinine, rat poison strychnine, and anti-hypertensive reserpine. In addition to these, over a dozen other MIA's have been identified as having important biological activities (Table 1). The most significant and well-known MIA's however have been the anti-cancer drugs vinblastine (VBL) and vincristine (VCR). First isolated in 1958 by the Canadian chemists Robert Noble and Charles Beer from the leaves of Catharanthus Roseus, these drugs were found to be potent inhibitors of microtubule formation and were the first natural products used to treat cancer [2]. The discovery provided a scientific explanation for the medicinal properties of $C$. roseus, which had a long history of medical folklore. Vinblastine and vincristine have since been proven to be effective treatments for Hodgkin's lymphoma, lung cancer, bladder cancer, brain cancer, testicular cancer and many others diseases [3-5] Both molecules are listed on the WHO's list of modern essential medicines [6], and are considered a key part of a modern health care system. For this reason, the production of MIA's such as VBL and VBC is an area of great scientific and commercial interest. 
Table 1: List of pharmaceutically active MIA's and their functions

\begin{tabular}{|c|c|c|c|}
\hline Alkaloid & Known For & Plant source & Reference \\
\hline Vinblastine & Anti-Cancer & C.roseus & {$[2]$} \\
\hline Vincristine & Anti-Cancer & C.roseus & {$[2]$} \\
\hline Ajmalicine & Anti-hypertensive & C.roseus, R.serpentina & [7] \\
\hline Alstonine & Anti-psychotic & C.roseus, A.Boonei & [8] \\
\hline Camptothecine & Antineoplastic & C. acuminata & [9] \\
\hline Ellipticine & Anti-Cancer & $\begin{array}{l}\text { O. borbonica, E. } \\
\text { coccinea }\end{array}$ & {$[10]$} \\
\hline Emetine & $\begin{array}{l}\text { Anti-protozoal, } \\
\text { emetic }\end{array}$ & $\begin{array}{l}\text { C. ipecacuanha, } P \text {. } \\
\text { ipecacuanhau }\end{array}$ & {$[11]$} \\
\hline Quinidine & Anti-arrhythmic agent & C. ledgeriana & {$[12]$} \\
\hline Quinine & Anti-malarial & C. ledgeriana & {$[13]$} \\
\hline Rescinnamine & Anti-hypertensive & R. serpentina & {$[14]$} \\
\hline Reserpine & $\begin{array}{l}\text { Anti-psychotic, anti- } \\
\text { hypertensive }\end{array}$ & $R$. serpentina & {$[15]$} \\
\hline Toxiferine & Muscle relaxant & S. toxifera & {$[16]$} \\
\hline Vincamine & Vasodilator & $V$. minor & [17] \\
\hline Yohombine & $\begin{array}{l}\text { Monoamine oxidase } \\
\text { inhibitor, aphrodisiac }\end{array}$ & P. yohimbe & {$[18]$} \\
\hline
\end{tabular}




\subsection{Production Strategies and Economics of the Monoterpenoid Indole Alkaloids}

Current production processes for VBC, VBL and most other MIA's rely almost entirely on the mass cultivation of $C$. roseus. After the plant has reached maturity, the leaves are mechanically harvested, pooled and prepared for extraction. Several extraction methods have been explored including centrifugal partition chromatography, supercritical $\mathrm{CO}_{2}$ extraction [19], negative pressure cavitation [20] and ultrasound assisted extraction [21]. The central problem with $C$. roseus-based alkaloid production is that in planta levels of dimeric bis-indole alkaloids are extremely low, usually in the range of 0.01-0.1 $\mathrm{mg} / \mathrm{gDCW}$ [22]. At these concentrations, a single gram of vinblastine requires over $500 \mathrm{~kg}$ of $C$. roseus leaves to produce [23], and costs anywhere from 770-3170\$/g [24]. The overall process is costly, inefficient, and extremely laborious. The low yield and high production cost of these drugs is a major constraint on their use worldwide, and is a problem that needs to be urgently addressed. This demand has spurred research into alternate production strategies including direct chemical synthesis, in vitro cell culturing, and metabolic engineering of $C$. roseus. The next section of this thesis will review the progress of these methods with respect to $\mathrm{VBL}$ and $\mathrm{VBC}$ production. Although impressive technical advances have been made in all three areas, none have found significant use as a large-scale MIA production platform.

\subsubsection{Chemical Synthesis of MIA's}

Chemical synthesis of VBL and VCR could provide an ideal solution to production problems inherent in C. roseus as it side-steps the use of plant biomass. A major technical challenge to this approach however is in the high entantio-structural complexity of the dimeric bis-indole alkaloids (Figure 1). There are 7 chiral centers on vinblastine and 9 chiral centers on vincristine, and any synthetic approach must be careful to maintain this chirality. The general method underlying most chemical synthesis strategies is to couple 
monomeric MIA's together, and then functionalize the resulting products. In the case of vinblastine, vindoline and catharanthine are coupled to produce anhdrovinblastine, which is then oxidized to give vinblastine with an overall yield of $40 \%[25,26]$ The reaction is not perfectly stereospecific and there is a $20 \%$ loss to leurosidine, a vinblastine regioisomer [26]. In the case of vincristine there is a slight variation, and a modified version of vindoline ( $\mathrm{N}$-desmethylvindoline) used in its place to produce $\mathrm{N}$ desmethylvinblastine. This product is then formylated to vincristine with a $39 \%$ overall yield [25]. While these methods are efficient, they face the major limitation that they rely on monomeric MIA's such as vindoline and catharanthine as precursors. These molecules are also exceptionally complex, and are currently only produced using $C$. roseus. While they are found in higher quantities than the dimeric bis-indole alkaloids $(2.08 \pm 113 \mathrm{mg} / \mathrm{g}$ DW for vindoline and $2.90 \pm 384 \mathrm{mg} / \mathrm{g}$ DW for catharanthine)[27], their availability is still limited by plant production. Although chemical synthesis might provide added value for monomeric MIA's extracted alongside VCR and VBL, the process is unlike to supplant $C$. roseus as a primary production source.
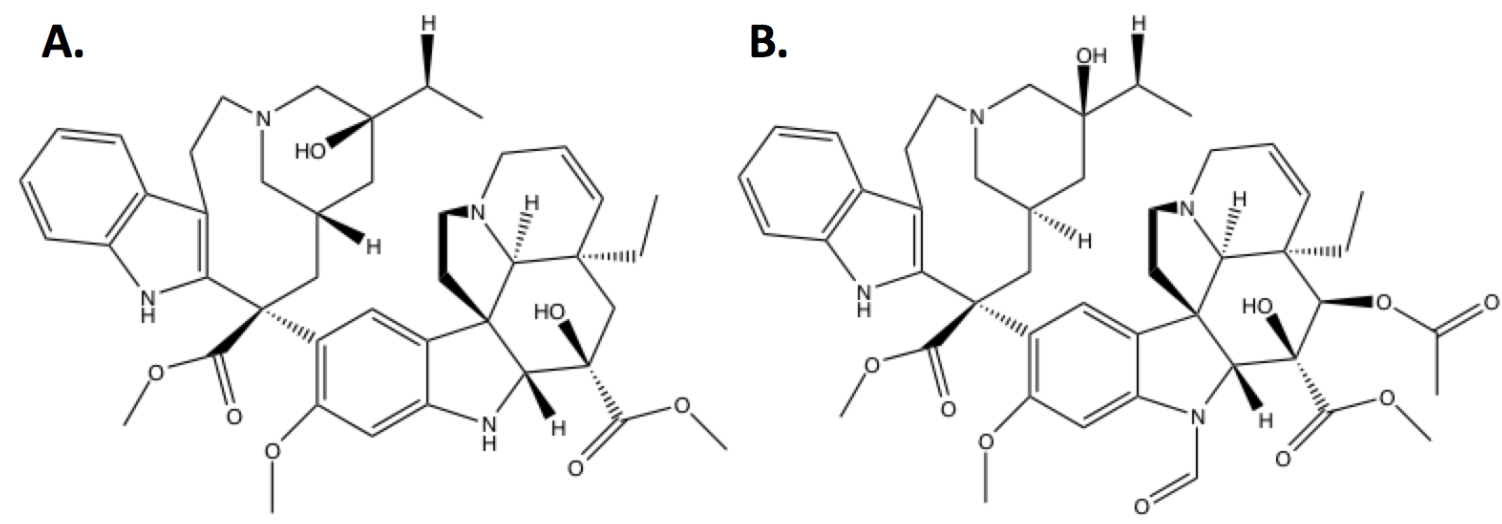

Figure 1: Chemical Structure of a) Vinblastine b) Vincristine 


\subsubsection{Metabolic Engineering of $C$. roseus}

Another potential way to improve MIA production is by metabolic engineering C. roseus directly. This strategy has the advantage of working in a system already validated for MIA production and even minor improvements in VBL or VBC yields can greatly improve the process economics. $C$. roseus has been proven to be tractable using techniques such as A. tumefaciens mediated gene transfer [28] and particle bombardment [29]. When the gene deacetylvindoline-4-O-acetyltransferase was transformed to $C$. roseus an increase in vindoline was observed from $1.15 \mathrm{mg} / \mathrm{g}$ in the wild type to 1.42 $2.7 \mathrm{mg} / \mathrm{g}$ in the engineered plants [30]. Another group successfully overexpressed both the MIA transcription factor ORCA3 and the geraniol-10 hydroxylase gene [31]. This increased the levels of a number of important metabolites such as vindoline $(1.25-3 \mathrm{mg} / \mathrm{g}$ vs $0.7 \mathrm{mg} / \mathrm{g}$ in control lines) and catharanthine, $(3.65-5.7 \mathrm{mg} / \mathrm{g}$ vs. $1.99 \mathrm{mg} / \mathrm{g}$ in control lines). Levels of the dimeric MIA's were relatively unchanged however with $0.07 \mathrm{mg} / \mathrm{g}$ vinblastine for the engineered plants vs. $0.05 \mathrm{~m} / \mathrm{g}$ in the control. While promising in theory, attempts to genetically manipulate $C$. roseus have generally failed to improve levels of dimeric bis-indole alkaloids such as VBL and VBC. The complexity of the

pathway, alongside the complex spatial and environmental regulation of MIA's in vivo makes it difficult to determine what the appropriate metabolic engineering strategy should be. Furthermore, the process is hampered by the long growth times and clumsy transformation techniques inherent in working with C. roseus. In some cases, transgenic C. roseus lines have been reported to be unstable, with the wildtype phenotype reemerging after successive generations [32]. These difficulties generally make the idea of improving MIA production by transgenic C. roseus cultivars unappealing. 


\subsubsection{In vitro Cell Cultures for MIA Production}

Growing cultures of C.roseus in bioreactors is another potential way of improving the MIA production process. Plant cell cultures have quicker development times, less variation in product yield and quantity, and are easier to apply good manufacturing practices (GMP) to than whole plant based processes [33]. While this method is generally more capital intensive than plant production [34], several strategies can be employed to boost MIA yields within cell cultures. These include screening of high MIA producing cultivars, optimization of media and culture conditions and employing different feeding and elicitation techniques. In many cases this approach has been highly successful. Suspension cultures have achieved up to $200 \mathrm{mg} / \mathrm{L}$ of adjmaline and $155 \mathrm{mg} / \mathrm{L}$ of catharanthine which corresponds to a 40 and 31 fold improvement vs. the plant respectively [35]. One major drawback to this method is that it is difficult to produce more complex MIA's such as the dimeric bis-indole alkaloids VBC and VBL. This is because their biosynthesis is a highly coordinated process in planta, and involves multiple cell types. While VCR and VBL ultimately accumulate in the epidermal latificer cells, the pathway spans at least four different cell types and much of the specifics behind the spatial organization is unknown [36]. This makes it difficult to produce dimeric bisindole alkaloids unless co-cultures or precursor feedings are employed.

\subsubsection{Summary of Production Systems for the Monoterpene Indole Alkaloid's}

Production of MIA's using C. roseus is an expensive and inefficient process and alternative approaches have so far been unable to solve this problem. C. roseus is too complex and slow growing to engineer efficiently, and transgenic cultivars can be genetically unstable. VBC and VBL can be synthesized chemically, but these procedures are ultimately dependent on monomeric MIA's, which themselves must be extracted from C. roseus. In vitro plant cultures can provide sources for monomeric MIA's, but the 
distribution of the pathway across multiple cell types makes the production of compounds like VBL and VCR difficult. A logical solution to this problem would be to reconstruct this pathway in a more tractable host such as Saccharomyces cerevisiae. Genetically modified yeast would provide an efficient, scalable, cost effective way of producing critical MIA's. Furthermore, an MIA-producing platform S. cerevisiae strain could be customized to facilitate exploration of a large alkaloid structural space, which could potentially lead to the discovery of new pharmaceuticals. Yeast already has a successful track record in this area, being used to produce valuable natural product-sourced pharmaceuticals such as the anti-malarial artemisinin [37] and more recently the benzylisoquinoline alkaloids [38-40]

\subsection{MIA Biosynthesis and Pathway}

\subsubsection{Overview}

The MIA pathway is highly complex and poorly understood. While there are parts of the pathway that are well characterized, such as biosynthesis of geraniol, tryptamine, as well as early Iridiod biosynthesis, there are large gaps in our knowledge of the enzymatic synthesis of even basic MIA monomers such as vindoline and catharanthine. While there are still many things that remain unknown about the MIA biosynthetic pathway, the field has advanced enough to allow for significant groundwork to be done in reconstructing the pathway in yeast. The discovery of several genes the in the early Iridiod pathway have opened the possibility of creating strictosidine producing strains of yeast de novo $[22,41$, $42,43]$. As strictosidine is the universal precursor for all MIA's, this would be extremely valuable. The next section of this work will review the biochemistry of the pathway as it relates to potential for strain engineering. For simplicity, it can be divided into four parts: 1) the shikimate pathway, producing the indole subunit tryptamine, 2) the mevalonate pathway producing the terpene geranyl pyrophosphate, 3) the "early" MIA pathway covering up the biosynthesis of strictosidine and, 4) the late MIA pathway covering the 
steps from strictosidine to $\mathrm{VBL} / \mathrm{VBC}$.

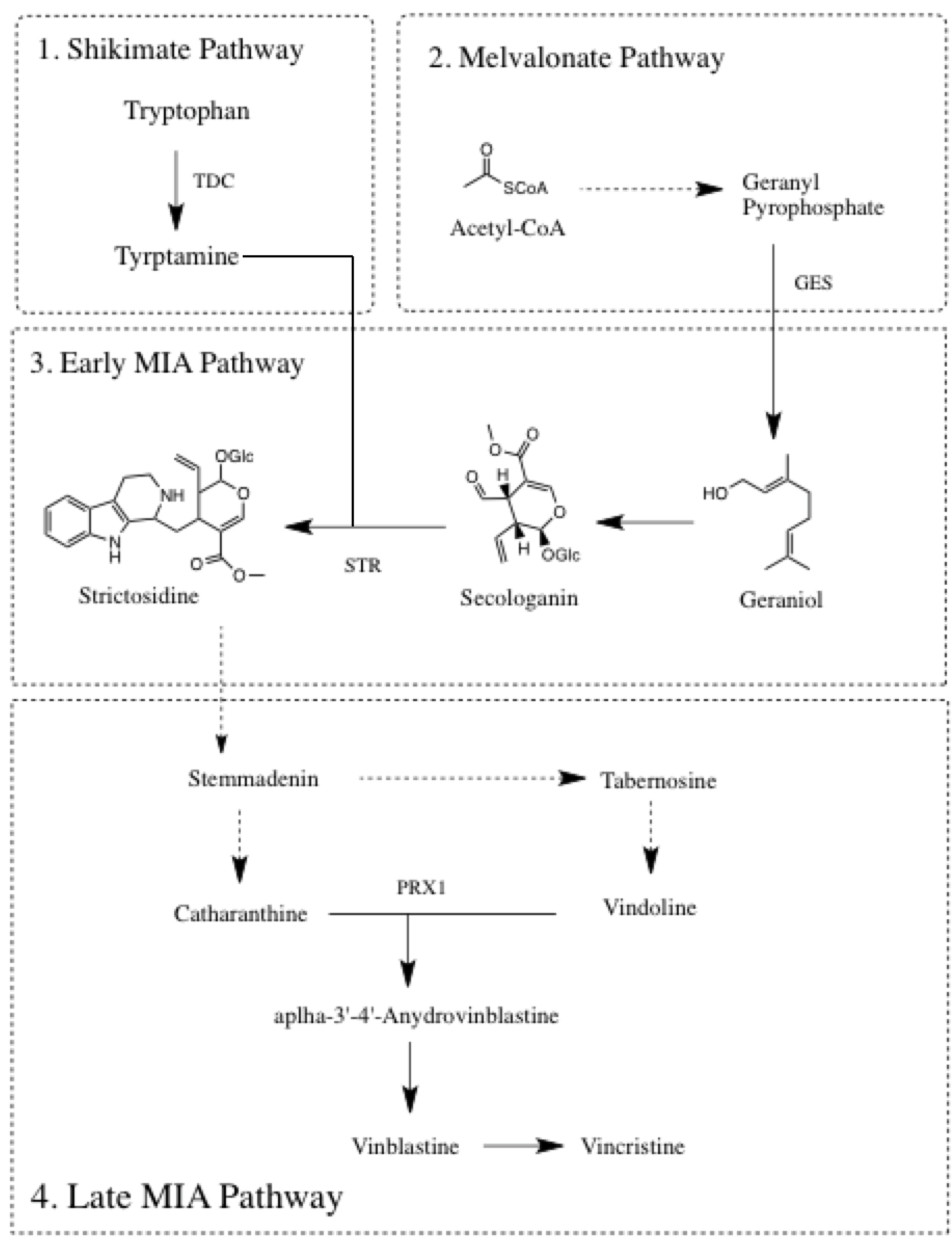

Figure 2: Overview of the MIA Pathway. The general pathway can be divided into four parts: 1) The shikimate pathway leading to the biosynthesis of tryptamine. 2) The 
mevalonate pathway from acetyl-coA to geranyl-pyrophosphate. 3) The "early" MIA pathway from geraniol to the universal MIA precursor strictosidine. 4) The "late" MIA pathway from strictosidine to VBL and VCR. Abbreviations: GES, geraniol synthase; TDC, tryptophan decarboxylase; STR, strictosidine synthase; PRX1, peroxidase 1.

\subsubsection{The Shikimate Pathway}

The shikimate and mevalonate pathways provide the respective terpene and indole portions of MIA pathway, and are the starting points of the biosynthetic process. The shikimate pathway is common to bacteria, fungi and algae is required to produce the aromatic amino acids phenylalanine, tyrosine and tryptophan [44]. It begins using the glycolytic products PEP and E4P, which condense to DAHP through DAHP synthase. From here, a series of 6 additional enzymatic reactions are needed to produce chorismate, which is the common precursor to the aromatic products of this pathway. Chorosimate can be converted to anthranilate by anthranilate synthase, which is then ribosylated by phosphoribosyl diphosphate PR-anthranilate transferase. This molecule is then isomerized by PR-anthranilate isomerase to create 1-(O-carboxyphenylamino)-1deoxyribulose phosphate. Partial cyclization by indole-3-glycerol phosphate synthase yields indole-3-glycerol phosphate. The two enzymes tryptophan synthase $\propto$ and $\beta$ synthase act on this molecule to form tryptophan through an indole intermediate. The last step to create tryptamine is the expression of a tryptophan decarboxylase (TDC), which is not native to yeast but has been well characterized in C. roseus [45]. As yeast is a native tryptophan producer no genetic modifications other than the expression of TDC are needed to form the indole precursor tryptamine.

\subsubsection{The Mevalonate Pathway}

The biosynthesis of GPP proceeds by the mevalonate pathway (Figure 3), which is common to all eukaryotes, archae and some bacteria [46]. This pathway is essential to 
yeast cellular viability and is responsible for the production of important sterols and polyisoprenoids such as squalene and ergosterol. It begins with the condensation of two acetyl-CoA's by the enzyme acetyl-CoA thiolase to form aceto-acetyl-CoA. An additional acetyl-CoA is added by acetyl-coA acetyltransferase (ERG10) to create 3hydroxymethylglutaryl-CoA (HMG-CoA). This molecule is toxic, and is an important natural bottleneck in the pathway [47]. A reduction of HMG-CoA by the enzyme hydroxymethylglutaryl-coA synthase (ERG13) gives mevalonate, which undergoes a series of phosphorylations, first by mevalonate kinase (ERG12), then by phosphomevalonate kinase (ERG8) to form mevalonate pyrophosphate. This can then be decarboxylated by mevalonate decarboxylase (Mvd1) to create isopentyl pyrophosphate. IPP is the basic building block for all terpinoids and can be isomerized to dimethylallyphosphate (DMAPP) by IPP isomerase (IDI). As a final step in monoterpene biosynthesis, DMAPP and IPP condense to form GPP by the action of farnesyl pyrophosphate synthase (ERG20). This enzyme can also catalyze an additional reaction of GPP with DMAPP to create the base sesquiterpene farnesyl-pyrophosphate (FPP). The pathway is regulated at the molecular level, and the pyrophosphates GPP and FPP both cause feedback inhibition to mevalonate kinase, which can lead to elevated levels of intracellular mevalonate [48]. While the MIA monoterpene precursor GPP is naturally produced by yeast, it is quickly converted to FPP and most strains of yeast do not produce monoterpenes [49]. Therefore, the first step in producing MIA's in yeast should be engineering a strain that can accommodate high titers of monoterpenes. 


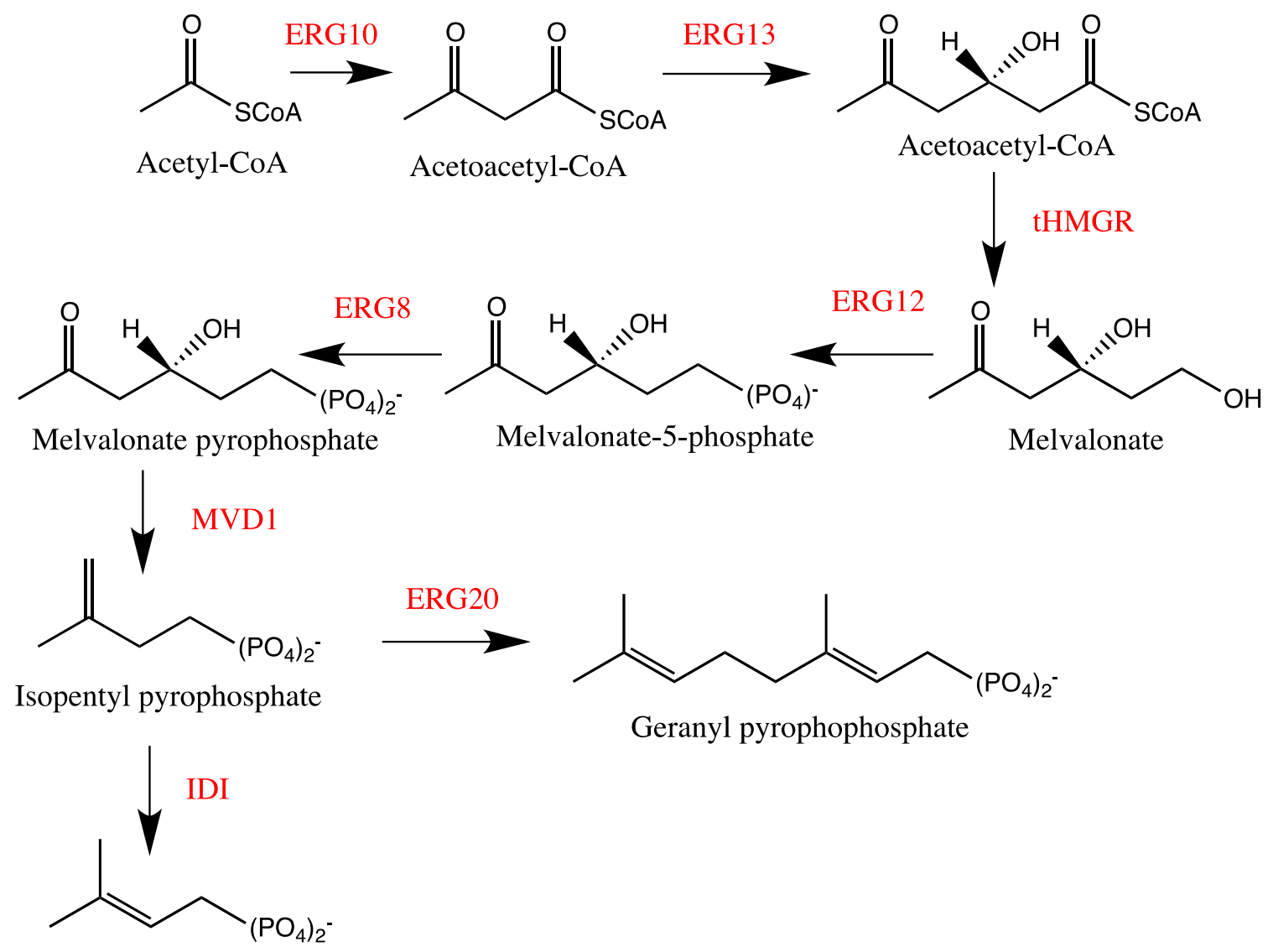

Melvalonate pyrophosphate

Figure 3: The mevalonate pathway from S. cerevisiae. Abbreviations: ERG10, acetoacetyl-coA thiolase; ERG13, 3-hydroxy-3-methylglutaryl-CoA synthase; tHMGr, 3hydroxy-3-methylglutaryl-CoA (HMG-CoA) reductase; ERG12, mevalonate kinase; ERG 8, phosphomevalonate kinase; MVD1; mevalonate decarboxylase; IDI, isopentyl pyrophosphate isomerase; ERG20, farnesyl pyrophosphate synthetase. 
Genetically modifying yeast to produce monoterpenes has been explored previously and has a wide range of applications from improving the organoleptic properties of white wines [49] to producing biofuels [50]. This can be achieved by overexpressing enzymes of the mevalonate pathway[37,51] and by introducing variants of the native yeast ERG20 enzyme that impairs its ability to bind GPP and convert it to FPP [52]. FPP is an essential metabolite that leads to sterol synthesis in yeast but is an undesirable product when optimizing GPP synthesis. Alternatively, there are plant analogs of Erg20 such as the Gpps2 enzyme from Abies grandis that produce GPP exclusively [53]. Other methods focus on increasing the global terpene supply such as overexpressing the tRNA regulator MAF1 to reduce loss of IPP to tRNA [54]. Additionally, the mutant transcription factor UPC2-1 can be expressed to globally up regulate the genes involved in sterol biosynthesis [55]. By using these methods, it has been demonstrated that yeast is capable of making a wide range of monoterpenoids such as linalool $(95 \mu \mathrm{g} / \mathrm{L})$ [56], geraniol (36.04 mg/L) [57], limonene $(0.49 \mathrm{mg} / \mathrm{L})$ [58] at high titers.

\subsubsection{The MIA Pathway to Strictosidine}

The "early" pathway begins at geraniol and extends to the universal MIA precursor strictosidine (Figure 4). The process starts with the hydroxylation of geraniol by the cytochrome P450 G10H and it's associated reductase CPR [59]. After hydroxylation, 10hydroxygeraniol undergoes two successive oxidations by the enzyme 10-hydroxygeraniol oxidase (10HGO) to form 10-oxogeraniol [60]. This is then cyclized by the reductive cyclase Iridiod synthase (IS) to form nepetalactol, which exists in chemical equilibrium with the dialdehyde iridodial. Nepetalactol is further oxidized by the enzyme 7deoxyloganic acid synthase (7DLS) to form 7-deoxyloganetic acid. This is glycolsated by 7-dexoxyloganetic acid glucosyl transferase (7DGLT), hydroxylated by 7-deoxyloganic acid hydroxylase (7DLH) and methylated by loganic acid O-methyltransferase (LAMT) to form loganin. Finally, the cyclopentane ring in loganin is cleaved by the enzyme secologanin synthase (SLS) to form secologanin, which condenses with L-trypatime via 
the enzyme strictosidine synthase (STS) to create strictosidine.
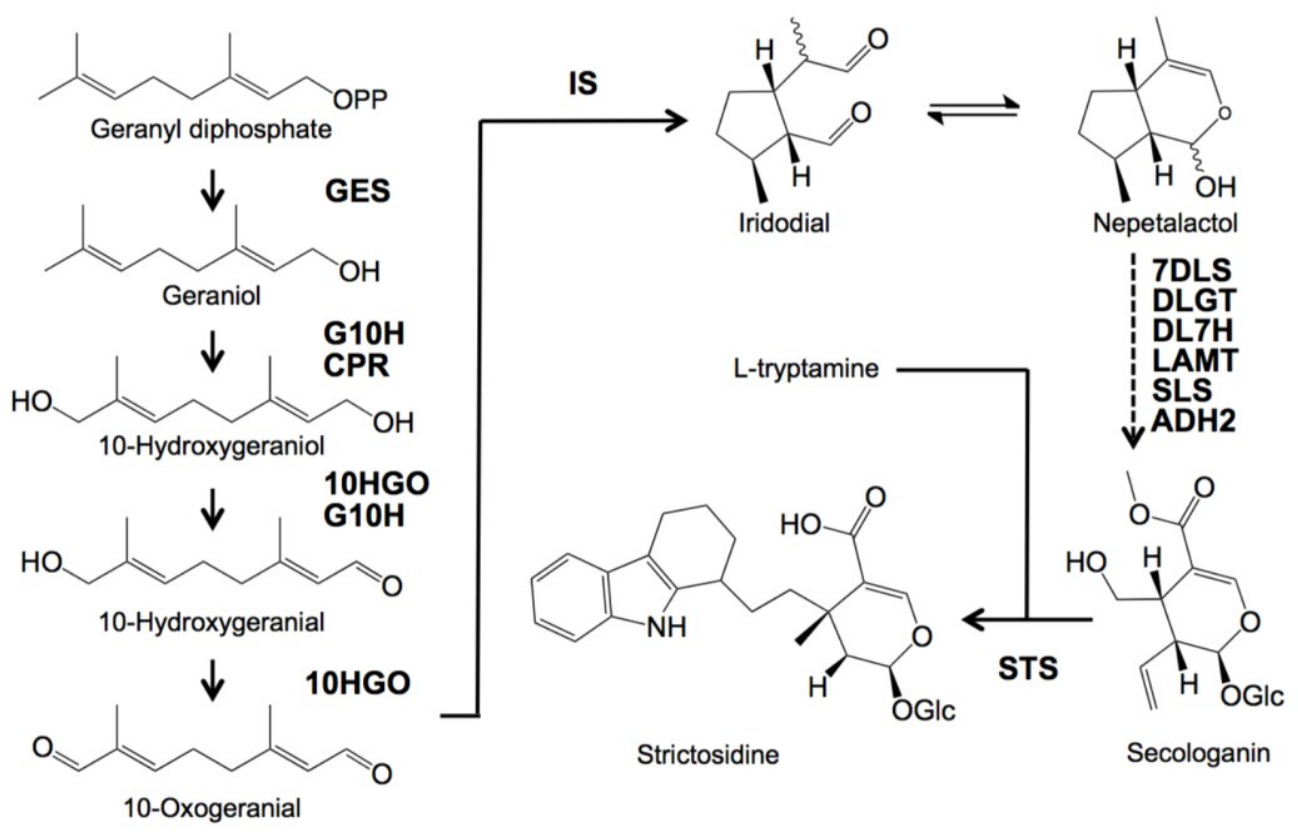

Figure 4: The "early" MIA pathway from GPP to strictosidine. From the mevalonate pathway intermediate GPP, a total of eleven genes are necessary to reach strictosidine. Abbreviations: GES, geraniol synthase; G10H, geraniol 10-hydroxylase; CPR, cytochrome P450 reductase; 10HGO, 10-hydroxygeraniol oxidoreductase; IS, Iridiod synthase; 7DLS, 7-deoxyloganic acid synthase; DLGT, 7-dexoxyloganetic acid glucosyl transferase; 7DLH, 7-deoxyloganic acid hydroxylase; LAMT, loganic acid Omethyltransferase; SLS, secologanin synthase; STR, strictosidine synthase; ADH2, alcohol dehydrogenase 2 . 


\subsubsection{The MIA Pathway to Vinblastine/Vincristine}

The reactions after strictosidine, referred to here as the "late MIA pathway" are ultimately responsible for creating commercially valuable MIA's such as VBL, VBC and adjmaline (Figure 5). A major limitation in reconstructing the entire MIA pathway in yeast is that many steps in this pathway remain unknown, or are entirely hypothetical. This section will review the parts that are known, and how they relate to the goal of biosynthesizing high value MIA's in S.cerevisiae. The first step in the synthesis of VBL or $\mathrm{VBC}$ is the deglycolysation of strictosidine to create cathenamine by the enzyme strictosidine beta-glucosidase [46]. A cathenamine reductase can then be used to form the anti-arrhythmic adjmaline, which the most easily accessible MIA that is commercially valuable. Cathenamine also exists in chemical equilibrium with 4,21dehydrogroissoschizine, and this is thought to continue down the pathway to form stemmadeniene [62], although the exact enzymatic process is unknown. Stemmadeniene, when fed to C. roseus suspensions cultures, produces the important MIA's catharanthine and tabernosine [63], though again the exact steps are unknown. The pathway is better understood after this point and tabernosine undergoes conversion to vindoline through a series of 8 enzymatic reactions [64]. This section of the pathway was recently reconstructed in yeast and the resulting strains were able to convert fed tabernosine to vindoline with a 7.5\% efficiency [64]. Vindoline and catharanthine are then coupled together by a peroxidase to form the highly unstable dihydropyroiminium, which spontaneously rearranges to $\propto-3^{\prime}, 4$-anhydrovinblastine [65]. Finally, this molecule can be hydroxylated across the double bond to form vinblastine or the N-methyl groups can be oxidized to yield vinblastine. 


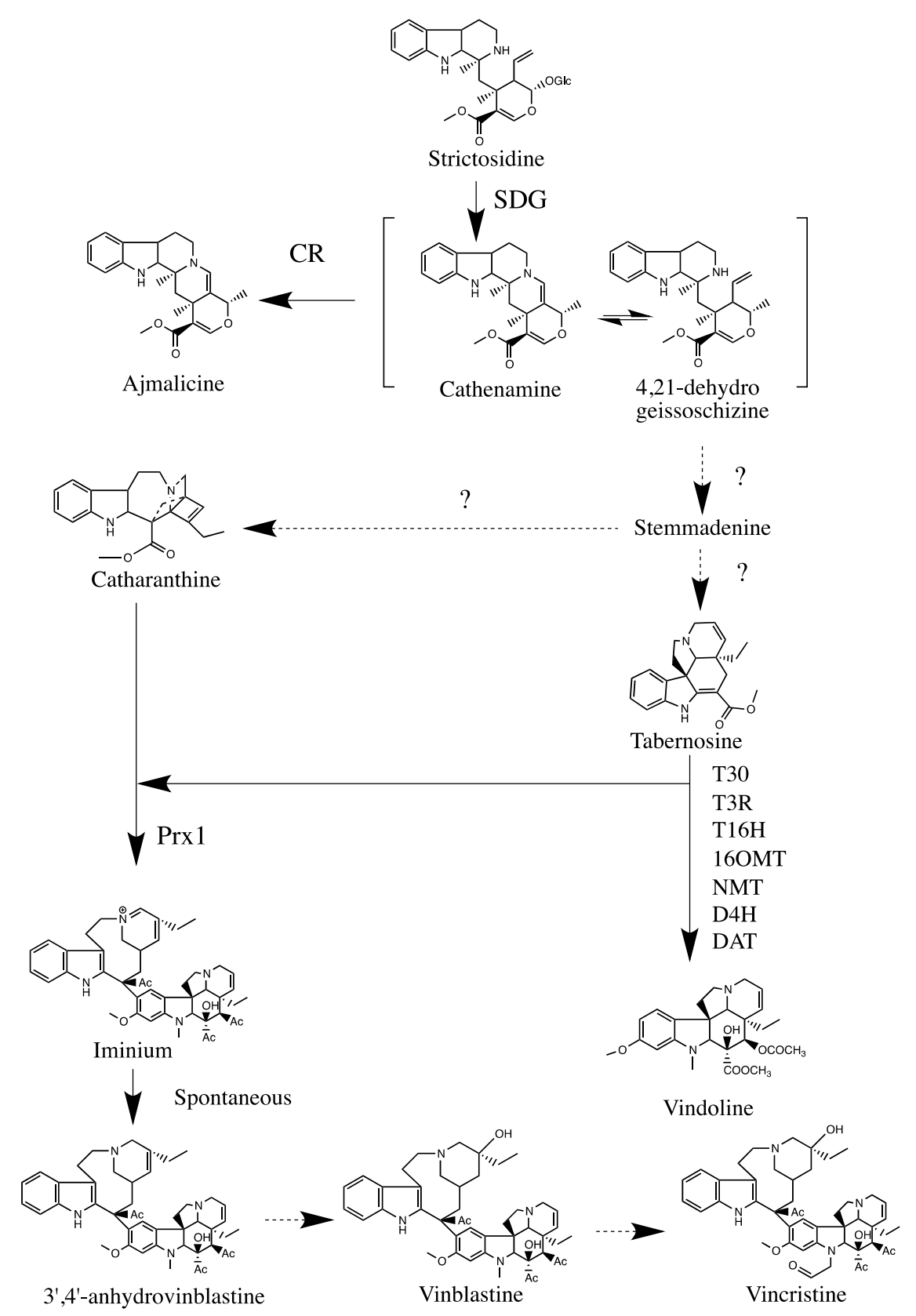

Figure 5: The MIA pathway from strictosidine to Vinblastine/Vincristine.

Abbreviations: SDG, strictosidine beta-D-glucosidase; CR, cathenamine reductase; T30, tabernosine 3-oxygenase; T3R, tabernosine 3 reductase; T16H, tabernosine 16 hydroxylase; 16OMT, 16-hydroxytabersonine O-methyltransferase; NMT, 3-hydroxy-16methoxy-2,3-dihydrotabersonine N-methyltransferase; D4H, desacetoxyvindoline-4hydroxylase; DAT, deacetylvindoline-4-O-acetyltransferase 


\subsection{General Reconstruction Strategy}

While VBR and VBL are ultimately the most valuable MIA's, they make poor initial synthesis targets due both to their complexity and the lack of information surrounding the intermediate enzymatic processes. Strictosidine is a more logical initial goal as all the enzymes required for its formation have been discovered and it is the common MIA precursor. By integrating all of the enzymes in the early MIA pathway in a GPP overproducing strain, strictosidine biosynthesis was recently achieved in yeast at low yields [66]. Although this is in important technical advancement, higher titers will be needed to reach downstream products like VBL and VBC, which are 21-23 additional enzymatic steps from strictosidine. In this thesis we report on the engineering of a nepetalactol-producing yeast strain as a first step to optimizing strictosidine biosynthesis in S. cerevisiae. The work was done in a two-stage process (Figure 6). First we optimized production of the base monoterpene geraniol using a combination of MVP enzyme overexpression and integration of an ERG20 $0^{\mathrm{K} 197 \mathrm{E}}$ mutation. Next, we characterized the downstream enzymes to nepetalactol and showed 10-hydroxygeraniol production in vivo as well as nepetalactol production in vitro. We also demonstrate that activity of the Iridiod synthase towards the intermediates geraniol and 10-hydroxygeraniol results in the synthesis of the non-productive intermediates citronellol and 10-hydroxycitronellol. This information will be vital to reconstitute and optimize an efficient strictosidine pathway in yeast. 


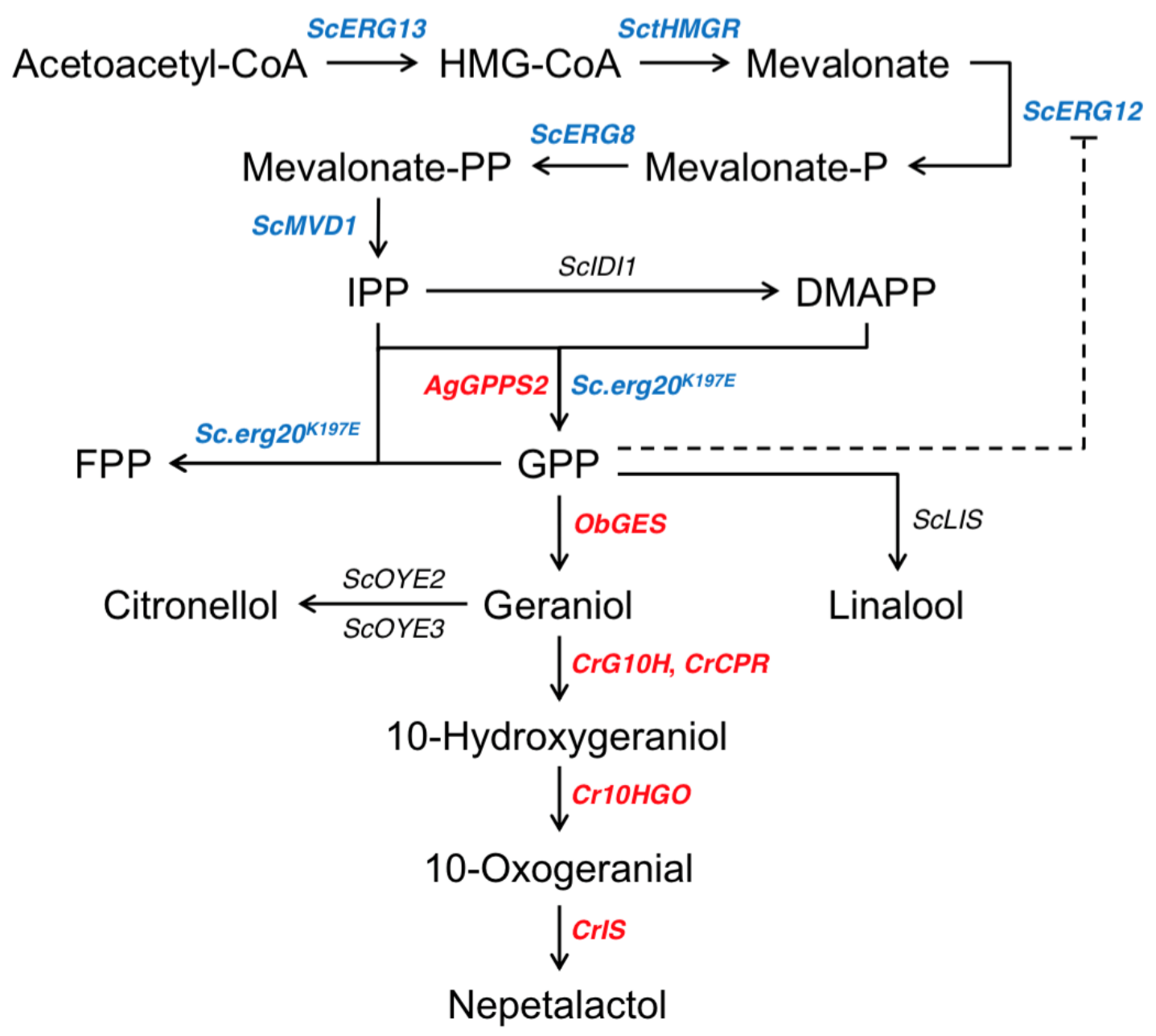

Figure 6: Metabolic engineering strategy to achieving nepetalactol synthesis in S.cerevisiae. Overexpressed yeast endogenous mevalonate pathway genes (blue) and plant genes (red) leading to nepetalactol. The endogenous ScERG20 gene was replaced with Sc.erg $20^{K 197 E}$ to decrease flux to FPP and improve GPP synthesis. Abbreviations: ScERG13, S. cerevisiae 3-hydroxy-3-methylglutaryl-CoA synthase; SctHMGR, S. cerevisiae 3-hydroxy-3-methylglutaryl-CoA reductase; Sc.ERG12, S. cerevisiae mevalonate kinase; ScERG8, S. cerevisiae phosphomevalonate kinase; ScMVD1 S. cerevisiae mevalonate pyrophosphate decarboxylase; ScIDII, S. cerevisiae isopentenyl pyrophosphate synthase; Sc.erg $20^{K 197 E}$, S. cerevisiae farnesyl pyrophosphate synthase variant; Ag.gpps, Abies grandis geranyl pyrophosphate synthase; ObGES, Ocimum basilicum geraniol synthase; $\mathrm{CrG10H}$, C. roseus geraniol 10-hydroxylase; C. roseus cytochrome P450 reductase; CrloHGO, C. roseus 10-hydroxygeraniol oxidoreductase; 
CrIS, C. roseus Iridiod synthase; ScLIS, S. cerevisiae linalool synthase (hypothesized, not yet identified), ScOYE2/3, S. cerevisiae NADPH dehydrogenase. Metabolites: IPP, isopentenyl pyrophosphate; DMAPP, dimethylallyl pyrophosphate; GPP, geranyl pyrophosphate; FPP, farnesyl pyrophosphate.

\subsection{Synthetic protein scaffolds}

The promiscuous activity of IS on geraniol was found to be a major barrier to reconstituting the MIA pathway in yeast. One potential way to fix this problem would be to use synthetic enzyme co-localization to physically sequester IS from the upstream enzymes. This can done using synthetic protein scaffolds, which can be highly effective at directing metabolic flux and improving overall pathway efficiency $[67,68]$. The basic structure of a protein scaffold is a set of modular protein binding domains strung together by flexible serine glycine linkers of the format (GGSG) $)_{x}$ (Figure 7). Peptides complementary to the binding domains are fused to enzymes in the desired pathway, which are then recruited to the scaffold. The domains used should be small, simple and have specific, tight binding ligands. Furthermore, they should not be dependent on ions, cofactors or post-translational modifications for function. Through a literature review, we have determined that the SH3 [69], GBD [70], SYNZIP1/2 [71], SYNZIP3/4 [71] and WW [72] domains would be appropriate candidates for a yeast scaffolding project. We have taken preliminary steps to characterizing the binding functionality of these domains in vivo. We also show that protein scaffolds can be used to improve metabolic flux within the MIA pathway. This makes them a useful tool for dealing with enzymatic spatial and regulatory problems that can occur when engineering long metabolic pathways in nonnative hosts. 


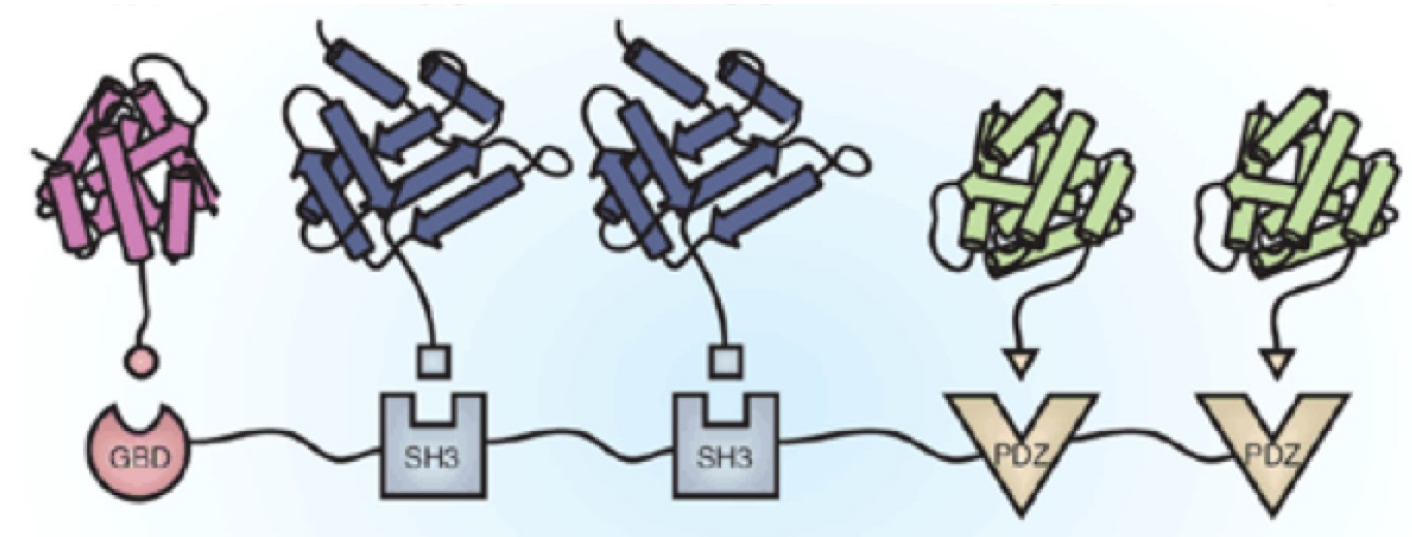

Figure 7: General structure of a synthetic protein scaffold: Scaffolds are created by fusing modular protein binding domains together by flexible glycine-serine linkers. Pathway enzymes are fused to peptides ligands containing binding the complementary domains, which are co-localized together on the scaffold. Adapted from [73].

\section{MATERIALS AND METHODS}

\subsection{Strains and plasmids}

Strains of $S$. cerevisiae used in this study and their genotype are listed in Table 2 . Escherichia coli DH5a was used to maintain and propagate plasmids. Escherichia coli was grown at $37^{\circ} \mathrm{C}$ and $200 \mathrm{rpm}$ in $\mathrm{LB}$ medium supplemented with $100 \mu \mathrm{g} / \mathrm{mL}$ of ampicillin. S. cerevisiae was grown at $30^{\circ} \mathrm{C}$ and $150 \mathrm{rpm}$ in either the rich medium YPD or the defined SD medium. When required, $200 \mu \mathrm{g} / \mathrm{mL}$ geneticin was added to YPD and SD medium was supplemented with amino acids to complement specific auxotrophic requirements.

All plasmids used in this study and the genes, promoters and terminators they contain are described in Table 2. Primers used in this study are listed in Table 3 The DNA assembler method [74] was used to construct the plasmids. The different DNA parts were amplified by PCR using Phusion High-Fidelity DNA polymerase (Thermo Fisher Scientific), 
resolved by gel electrophoresis and individually purified using Qiagen Gel Purification kit (Valencia, CA, USA). DNA parts (promoter, gene, terminator) with overlapping homologous sequences were pooled with a linearized plasmid and transformed into the appropriate auxotrophic yeast strain using either the Gietz method[75] or by electroporation, as described by Shao et al[76]. Assembled plasmids were selected by growth on minimal medium and the resulting plasmids were recovered from yeast and transformed into E. coli for maintenance. Sanger sequencing confirmed correct assembly of each construct. Promoters and terminators required for assembly were amplified from S. cerevisiae CEN.PK gDNA. Yeast mevalonate pathway genes ERG8, ERG12, ERG13 and a truncated $H M G R(t H M G R)$ were also amplified from $S$. cerevisiae CEN.PK gDNA and assembled together into either the high-copy shuttle vector pYES2 (Thermo Scientific) or a centromeric plasmid derived from pGREG505. Genes originating from plant species were assembled into similar versions of the multi-copy pYES(TRP) or pYES(URA). Geraniol synthase from Ocimum basilicum (ObGES) was assembled from synthesized gBlocks (Integrated DNA Technologies) based on the cDNA sequence (GenBank accession AY362553). Geraniol pyrophosphate synthase from Abies grandis (AgGPPS2) as well the cytochrome $\mathrm{P} 450$ reductase $(\mathrm{CrCPR})$, the geraniol 10hydroxylase $(\mathrm{Cr} G 10 H)$ and the 10-hydroxygeraniol oxidoreductase $(\mathrm{Cr} 10 \mathrm{HGO})$ from $\mathrm{C}$. roseus were codon optimized for expression in S. cerevisiae and synthesized by DNA 2.0 (GenBank accession to be submitted). The Iridiod synthase gene (GenBank accession JX974564) isolated from C. roseus cDNA by V. de Luca (Brock University) and cloned using primers 1060F and 1060R (Table 4). 
Table 2: Strains and plasmids used in this work

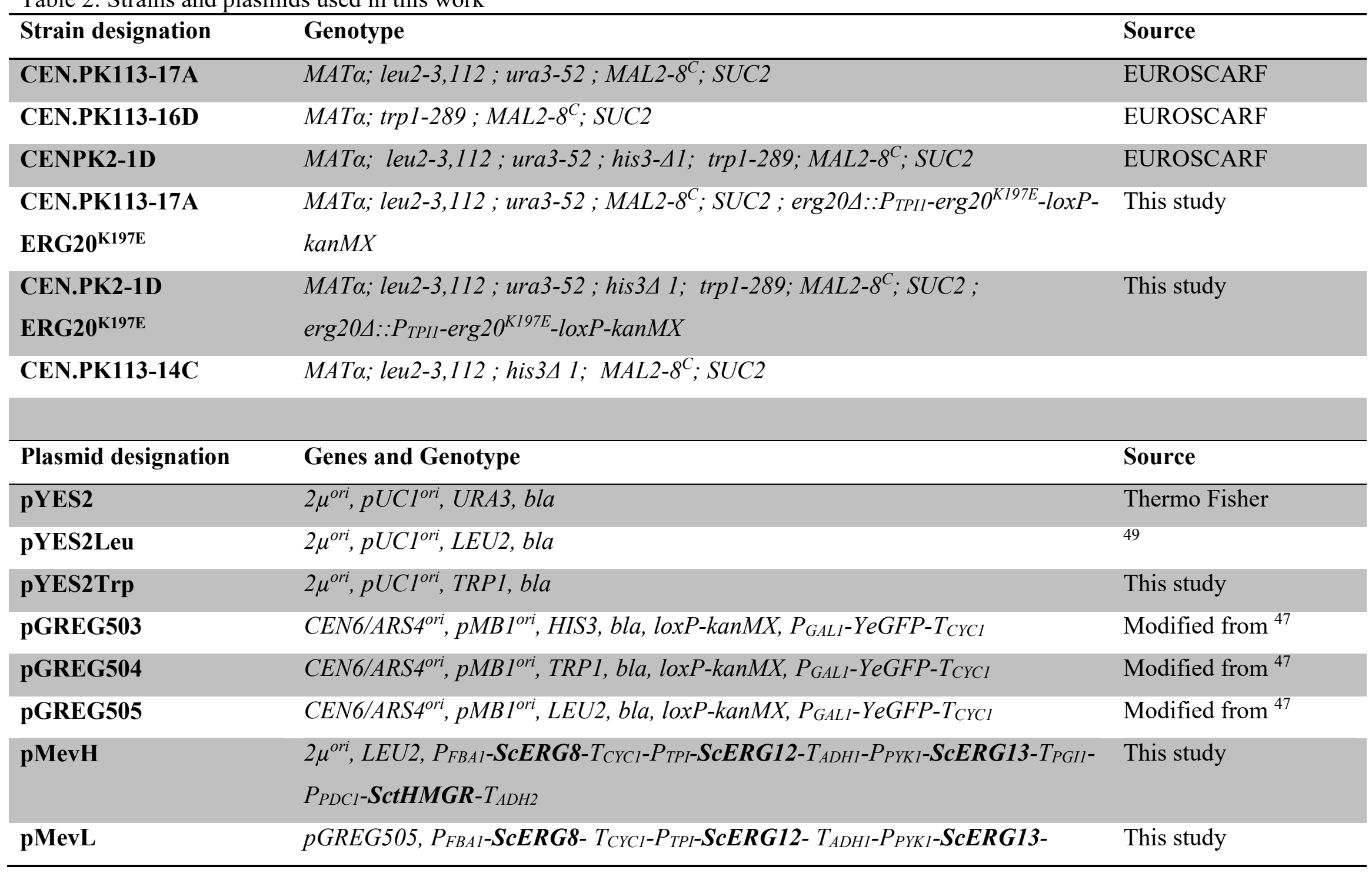




\begin{tabular}{|c|c|c|}
\hline & $T_{P G I 1}-P_{P D C 1}-\boldsymbol{S c t H M G R}-T_{A D H 2}$ & \\
\hline pGer & $2 \mu^{o r i}, U R A 3, P_{P Y K 1}-\boldsymbol{A g G P P S} 2-T_{E N O 2}-P_{P D C l}-\mathrm{CrCPR}-T_{A D H 2}-P_{T E F 2}-\mathrm{ObGES}-T_{T D H 2}$ & This study \\
\hline pGerOH & $\begin{array}{l}2 \mu^{o r i}, U R A 3, P_{P Y K 1}-\boldsymbol{A g G P P S} 2-T_{E N O 2}-P_{P D C 1}-\boldsymbol{C r C P R}-T_{A D H 2}-P_{T E F 2}-\mathrm{ObGES}-T_{T D H 2}- \\
P_{F B A 1}-\boldsymbol{C r G 1 0 H}-T_{P G I 1}\end{array}$ & This study \\
\hline pHGO & $2 \mu^{o r i}, T R P 1, P_{T E F I}-\mathrm{Cr} 10 \mathrm{HGO}-T_{P G I 1}$ & This study \\
\hline pHGOIS & $2 \mu^{o r i}, T R P 1, P_{T E F 1}-\mathrm{Cr} 10 \mathrm{HGO}-T_{P G H 1}-P_{T D H 3}-\mathrm{Cr} \boldsymbol{I S}-T_{A D H 2}$ & This study \\
\hline pIS & pGREG504, $P_{T D H 3}-\boldsymbol{C r} \boldsymbol{I S}-T_{A D H 2}$ & This study \\
\hline pNV-SH3 & pGREG503 $P_{T D H 3-N} \boldsymbol{V}-\mathbf{S H} 3-T_{A D H 2}$ & This study \\
\hline pCV-SH3 lig & pGREG505 $P_{\text {Teflp }}-\boldsymbol{C} \boldsymbol{V}-\mathbf{S H} 3_{\text {lig }}-T_{E N O 2}$ & This study \\
\hline pNV-GBG & pGREG503 $P_{T D H 3-N} \boldsymbol{V}-\mathbf{S H 3}-T_{A D H 2}$ & This study \\
\hline pCV-GBB lig & pGREG505 $P_{T e f 1 p}-\boldsymbol{C V}-\boldsymbol{G B} \boldsymbol{D}_{\text {lig }}-T_{E N O 2}$ & This study \\
\hline pNV-SYN1 & pGREG503 $P_{T D H 3}-\mathbf{N} \boldsymbol{V}-\boldsymbol{S Y N 1}-T_{A D H 2}$ & This study \\
\hline pCV-SYN2 & pGREG505 $P_{T e f 1 p}-\boldsymbol{C V}-\boldsymbol{S} Y \mathbf{N} 2-T_{E N O 2}$ & This study \\
\hline pNV-SYN3 & pGREG503 $P_{T D H 3-N V-S Y N 3-T_{A D H 2}}$ & This study \\
\hline pCV-SYN4 & pGREG505 $P_{\text {Teflp }}-\boldsymbol{C V}$-S YN4- $T_{E N O 2}$ & This study \\
\hline pNV-GHF & pGREG503 $P_{T D H 3-} \boldsymbol{N} \boldsymbol{V}-\boldsymbol{G H} \boldsymbol{F}-T_{A D H 2}$ & This study \\
\hline pCV-GHF iig & pGREG505 $P_{\text {Teflp }} \boldsymbol{C} \boldsymbol{V}$-GHF $\boldsymbol{F}_{\text {lig }}-T_{E N O 2}$ & This study \\
\hline $\mathbf{p N V}$ & pGREG503 $P_{T D H 3-N} \boldsymbol{V}-T_{A D H 2}$ & This study \\
\hline $\mathbf{p C V}$ & pGREG505 $P_{T e f 1 p}-\boldsymbol{C} \boldsymbol{V}-T_{E N O 2}$ & This study \\
\hline pVenus & pGREG503 $P_{T D H 3-\text { Venus- }} T_{A D H 2}$ & This study \\
\hline
\end{tabular}




\begin{tabular}{|c|c|c|}
\hline pERGGES & pGREG504 $P_{T D H 3}-\boldsymbol{E R G} 20^{\mathbf{K 1 9 7}}-\boldsymbol{S Y N 4}-T_{E N O 2^{-}} P_{T e f 1}-\boldsymbol{G E S}-\boldsymbol{G B G l i g -} T_{P G I}$ & This study \\
\hline pOYE2 & pGREG506 $P_{T P I-}$ OYE2-SH3lig- $T_{c y c}$ & This study \\
\hline pSYN3-(GBD)1-SH3 & pGREG503 $P_{T D H 3 p^{-}} \mathrm{SYN3}-(\mathrm{GBD})_{1}-\mathrm{SH} 3-T_{E N O 2}$ & This study \\
\hline pSYN3-(GBD)2-SH3 & pGREG503 $P_{T D H 3 p^{-}}$SYN3-(GBD)2-SH3- $T_{E N O 2}$ & This study \\
\hline pSYN3-(GBD)3-SH3 & pGREG503 $P_{T D H 3 p^{-}}$SYN3-(GBD) 3 -SH3- $T_{E N O 2}$ & This study \\
\hline
\end{tabular}

\section{Table 3: Oligonucleotides used in the work}

\begin{tabular}{|c|c|}
\hline Primer name & Sequence 5'-3' \\
\hline 506 FBA1p F & TAACCCTCACTAAAGGGAACAAAAGCTGGAGCTCGTTTAAACGGCGCGCCATCCAACTGGCACCGCTGGCTTG \\
\hline pYES FBA1pF & CGCTACAGGGCGCGTGGGGATGATCCACTAGTATCCAACTGGCACCGCTGGC \\
\hline ERG8 FBA1p R & CACTGAAGGCTCTCAACTCTGACATTGTTTTTATGTATTACTTGGTTATGGTTA \\
\hline FBA1p ERG8 F & TATAACCATAACCAAGTAATACATAAAAACAATGTCAGAGTTGAGAGCCTTCAGTGC \\
\hline CYC1t ERG8 R & AAGCGTGACATAACTAATTACATGATTATTTATCAAGATAAGTTTCCGGATC \\
\hline ERG8 CYC1t F & AAGATCCGGAAACTTATCTTGATAAATAATCATGTAATTAGTTATGTCACGC \\
\hline TPIp CYC1t R & TATCCGTAATCTTTAAACAGCTAGTGCAAATTAAAGCCTTCGAGCGTCCC \\
\hline CYC1t TPIp F & TTTTGGGACGCTCGAAGGCTTTAATTTGCACTAGCTGTTTAAAGATTACGGA \\
\hline ERG12 TPIp R & CGGTGCAGAAGTTAAGAACGGTAATGACATTGTTTTTTTATGTATGTGTTTTTTGTAGTTATAGATTTAAGC \\
\hline TPIp ERG12 F2 & TCTATAACTACAAAAAACACATACATAAAAAAACAATGTCATTACCGTTCTTAACTTCTG \\
\hline ADH1t ERG12 R & CTTGACCAAACCTCTGGCGAAGAAGTCCATTATGAAGTCCATGGTAAATTCGTGTTTCC \\
\hline ERG12 ADH1t F & CACGAATTTACCATGGACTTCATAATGGACTTCTTCGCCAGAGGTTTGGTC \\
\hline PYK1p ADH1t R & GAGATTAATCTCCAAAATAGTAGCATTGCATGCCGGTAGAGGTGTGGTCAAT \\
\hline ADH1t PYK1p F & CGCTCTTATTGACCACACCTCTACCGGCATGCAATGCTACTATTTTGGAGATT \\
\hline ERG13 PYK1p R & GTTTAGTTGAGAGTTTCATTGTTTTGATGTTTTATTTGTTTTGATTGGTGTC \\
\hline
\end{tabular}




\begin{tabular}{|c|c|}
\hline PYK1p ERG13 F & AAAACAAATAAAACATCAAAACAATGAAACTCTCAACTAAACTTTGTTGGTG \\
\hline PGIt ERG13 R & CTTTAGGTATATATTTAAGAGCGATTTGTTTTATTTTTTAACATCGTAAGATC \\
\hline ERG13 PGIt $\mathrm{F}$ & $\begin{array}{l}\text { AGAAGATCTTACGATGTTAAAAAATAAAACAAATCGCTCTTAAATATATACC } \\
\end{array}$ \\
\hline PDC1p PGIt R & $\begin{array}{l}\text { GCGGAACATATGCTCACCCAGTCGCATGTGGTATACTGGAGGCTTCATGAGT } \\
\end{array}$ \\
\hline PGIt PDC1p F & $\begin{array}{l}\text { GGACATAACTCATGAAGCCTCCAGTATACCACATGCGACTGGGTGAGCATAT } \\
\end{array}$ \\
\hline tHMGR PDC1p R & TTTATTGGTTAAAACCATTGTTTTTGATTTGACTGTGTTATTTTGCGTGAGG \\
\hline PDC1p tHMGR F & $\begin{array}{l}\text { AAATAACACAGTCAAATCAAAAACAATGGTTTTAACCAATAAAACAGTCATT } \\
\end{array}$ \\
\hline ADH2t tHMGR R & $\begin{array}{l}\text { AATCGTAAAGACATAAGAGATCCGCTTAGGATTTAATGCAGGTGACGGACC } \\
\end{array}$ \\
\hline tHMGR ADH2t F & $\begin{array}{l}\text { GGTCCGTCACCTGCATTAAATCCTAAGCGGATCTCTTATGTCTTTACGAT } \\
\end{array}$ \\
\hline $506 \mathrm{ADH} 2 \mathrm{t} \mathrm{R}$ & $\begin{array}{l}\text { ATAACTTCGTATAATGTATGCTATACGAAGTTATTAGGTACCGCGGCCGCTAGAATTATATAACTTGATGAGATGAG } \\
\end{array}$ \\
\hline pYES ADH2tR & $\begin{array}{l}\text { CGCGCGTTGGCCGATTCATTAATGCAGGGCCTAGAATTATATAACTTGATGAG } \\
\end{array}$ \\
\hline pYES PYK1p F2 & ACAGGGCGCGTGGGGATGATCCACTAGTAATGCTACTATTTTGGAGATTAA \\
\hline GPPS2 PYK1p R & $\begin{array}{l}\text { CCCATAGTAGCCATAGCAGAGTAAGCCATTGTTTTGATGTTTTATTTGTTTTGATTGGTG } \\
\end{array}$ \\
\hline PYK1p GPPS2 F & $\begin{array}{l}\text { CAAGACACCAATCAAAACAAATAAAACATCAAAACAATGGCTTACTCTGCTATGGCTACT } \\
\end{array}$ \\
\hline ENO2t GPPS2 R & AAAGACTAATAATTCTTAGTTAAAAGCACTTTAGTTTTGTCTGAATGCGACATAGTC \\
\hline GPPS2 ENO2t $\mathrm{F}$ & GCTGACTATGTCGCATTCAGACAAAACTAAAGTGCTTTTAACTAAGAATTATTAG \\
\hline TEF2p ENO2t R & $\begin{array}{l}\text { CTATATGTAAGTATACGGCCCCATATAAGGTATCATCTCCATCTCCCAT } \\
\end{array}$ \\
\hline ENO2t TEF2p F & $\begin{array}{l}\text { ATATGCATATGGGAGATGGAGATGATACCTTATATGGGGCCGTATACTTACATATAG } \\
\end{array}$ \\
\hline GES TEF2p R & $\begin{array}{l}\text { AACTTTCTGGCTTATGGATGACATTGTTTTTTAATTATAGTTCGTTGACCGTATATTCTAA } \\
\end{array}$ \\
\hline TEF2p GES F & TAGAATATACGGTCAACGAACTATAATTAAAAAACAATGTCATCCATAAGCCAGAAAGTT \\
\hline TDH2t GES R & CTAAATCATTAAAGTAACTTAAGGAGTTAAATTTAAACGTAAGGTTCGAACATCAACCCGCC \\
\hline GES TDH2t F & $\begin{array}{l}\text { GGGTTGATGTTCGAACCTTACGTTTAAATTTAACTCCTTAAGTTACTTTAATGATTTAG } \\
\end{array}$ \\
\hline PDC1p ENO2t R & GCGGAACATATGCTCACCCAGTCGCATGTAGGTATCATCTCCATCTCCCAT \\
\hline ENO2t PDC1pF & $\begin{array}{l}\text { ATATGCATATGGGAGATGGAGATGATACCTACATGCGACTGGGTGAGCATAT } \\
\end{array}$ \\
\hline FBA1p TDH2t R & GTTGTTCAAGCCAGCGGTGCCAGTTGGATGCGAAAAGCCAATTAGTGTGATAC \\
\hline TDH2t FBA1p F & AAAGCACTTAGTATCACACTAATTGGCTTTTCGCATCCAACTGGCACCGCTGGC \\
\hline G10H FBA1p R & $\begin{array}{l}\text { GTCAATATGATAGTTAAGTAATCCATTGTTTTTATGTATTACTTGGTTATGG } \\
\end{array}$ \\
\hline FBAlp G10H F & CATATATAACCATAACCAAGTAATACATAAAAACAATGGATTACTTAACTATCATATTGAC \\
\hline PGIt G10H R & $\begin{array}{l}\text { GCTTTAATGTTCTTTAGGTATATATTTAAGAGCGATTTGTTTCACAGGGTAGAAGGCACAGCTC } \\
\end{array}$ \\
\hline
\end{tabular}




\begin{tabular}{|c|c|}
\hline G10H PGIt F & CCTTTAAGAGCTGTGCCTTCTACCCTGTGAAACAAATCGCTCTTAAATATATACC \\
\hline CPR PDC1p R & $\begin{array}{l}\text { GGATAGTTTTTCTGAAGATGAGTCCATTGTTTTTGATTTGACTGTGTTATTTTGC } \\
\end{array}$ \\
\hline PDC1p CPR F & $\begin{array}{l}\text { GCAAAATAACACAGTCAAATCAAAAACAATGGACTCATCTTCAGAAAAACTATCC } \\
\end{array}$ \\
\hline ADH2t CPR R & $\begin{array}{l}\text { CGTAAAGACATAAGAGATCCGCCTACCAAACATCCCTTAAGTAACG } \\
\end{array}$ \\
\hline CPR ADH2t F & $\begin{array}{l}\text { CGTTACTTAAGGGATGTTTGGTAGGCGGATCTCTTATGTCTTTACG } \\
\end{array}$ \\
\hline ADH2t TEF2p F & $\begin{array}{l}\text { TTTACTCATCTCATCTCATCAAGTTATATAATTCTATATATGGGGCCGTATACTTACATA } \\
\end{array}$ \\
\hline 10HGO TEF1pR & $\begin{array}{l}\text { ATGTTCAACTTCTGGAGACTTGGCCATTGTTTTATTAAAACTTAGATTAGATTGCTATGC } \\
\end{array}$ \\
\hline TEF1p 10HGO F & $\begin{array}{l}\text { GAAAGCATAGCAATCTAATCTAAGTTTTAATAAAACAATGGCCAAGTCTCCAGAAGTTG } \\
\end{array}$ \\
\hline PGIlt 10HGO R & GTTCTTTAGGTATATATTTAAGAGCGATTTGTTTTAAGCAGACTTTAAGGTATTAGCG \\
\hline 10HGO PGI1t F & $\begin{array}{l}\text { TAATTGACGTCGCTAATACCTTAAAGTCTGCTTAAAACAAATCGCTCTTAAATATATACC } \\
\end{array}$ \\
\hline pYES TEF1p F & $\begin{array}{l}\text { ACAGGGCGCGTGGGGATGATCCACTAGTATAGCTTCAAAATGTTTCTACTCC } \\
\end{array}$ \\
\hline PGI1t TDH3p F & $\begin{array}{l}\text { CGAAGGACATAACTCATGAAGCCTCCAGTATACCTCGAGTTTATCATTATCAATACTGCC } \\
\end{array}$ \\
\hline IS TDH3p R & $\begin{array}{l}\text { CAGCACCAATGGACCTCTTCCACCACCAACTCATTGTTTTTCGAAACTAAGTTCTTGGTG } \\
\end{array}$ \\
\hline TDH3p IS F & $\begin{array}{l}\text { TTAGTTTTAAAACACCAAGAACTTAGTTTCGAAAAACAATGAGTTGGTGGTGGAAGAG } \\
\end{array}$ \\
\hline$\overline{\mathrm{ADH} 2 \mathrm{t} I \mathrm{IS} \mathrm{R}}$ & $\begin{array}{l}\text { TGAAAACTATAAATCGTAAAGACATAAGAGATCCGCCTAAGGAATAAACCTATAATCCCT } \\
\end{array}$ \\
\hline$\overline{I S ~ A D H 2 t ~} \mathrm{~F}$ & $\begin{array}{l}\text { TTGATAAGATGAGGGATTATAGGTTTATTCCTTAGGCGGATCTCTTATGTCTTTACG } \\
\end{array}$ \\
\hline pYES PG1t R & $\begin{array}{l}\text { CGCGCGTTGGCCGATTCATTAATGCAGGGCCCCGCGGCCGCGGTATACTGGAGGCTTCATGAG } \\
\end{array}$ \\
\hline$\overline{\mathrm{IS} F}$ & AGTTGGTGGTGGAAGAGGT \\
\hline IS R & AGGAATAAACCTATAATCCCTCAT \\
\hline C6506 R & $\begin{array}{l}\text { TAACCCTCACTAAAGGGAACAAAAGCTGGAGCTCGTTTAAACGGCGCGCCGAGACTGCAGCATTACTTTGAGAAG } \\
\end{array}$ \\
\hline C1506 F & $\begin{array}{l}\text { ATAACTTCGTATAATGTATGCTATACGAAGTTATTAGGTACCGCGGCCGCACAACTCATGGTGATGTGATTGCC } \\
\end{array}$ \\
\hline pYES TPIp F & $\begin{array}{l}\text { CCTGAATTCTTTTAGTTTATGTATGTG } \\
\end{array}$ \\
\hline pYES CYCt R & ATCATGTAATTAGTTATGTCACGC \\
\hline TPIp Erg20 F & $\begin{array}{l}\text { TCTTTTCTTGCTTAAATCTATAACTACAAAAAACACATACATAAACTAAAAAAAACAATGGCTTCAGAAAAAGAAATTAG } \\
\text { GAG }\end{array}$ \\
\hline CYCt Erg20 R & $\begin{array}{l}\text { GGATGTGGGGGGAGGGCGTGAATGTAAGCGTGACATAACTAATTACATGATCTATTTGCTTCTCTTGTAAACTTTGTTCAA } \\
\text { G }\end{array}$ \\
\hline pYES CYCt R & $\begin{array}{l}\text { GGCCCTGCATTAATGAATCGGCCAAC } \\
\end{array}$ \\
\hline pYES F $2^{\text {nd }}$ & GCAAATTAAAGCCTTCGAGCGTCC \\
\hline
\end{tabular}




\begin{tabular}{|c|c|}
\hline Kanx CYCt F & ACCTTGCTTGAGAAGGTTTTGGGACGCTCGAAGGCTTTAATTTGCCAGCTGAAGCTTCGTACGCTG \\
\hline Kanx pYES R & AAACCGCCTCTCCCCGCGCGTTGGCCGATTCATTAATGCAGGGCCGCATAGGCCACTAGTGGATCTG \\
\hline pYES CYC R $2^{\text {nd }}$ & GGCCCTGCATTAATGAATCGGCCAAC \\
\hline TDH3p NVenus F & TTTTTAGTTTTAAAACACCAAGAACTTAGTTTCGATCTAAAGGTGAAGAATTATTCACTG \\
\hline TDH3p NVenus R & CAACACCAGTGAATAATTCTTCACCTTTAGACATTGTTTTTCGAAACTAAGTTCTTGGTG \\
\hline NVenus Eno2 F & AATGTTTACATCACTGCTGACAAACAATAATAGAGTGCTTTTAACTAAGAATTATTAGTC \\
\hline NVenus-L1 R & $\begin{array}{l}\text { caatgtttacatcactgctgacaaacaaGGTGGAGGTTCTGGTGGAGGTAGTGGT } \\
\end{array}$ \\
\hline L1-SH3 F & GGTGGAGGTTCTGGTGGAGGTAGTGGTATGGAAGATTTGCCATTCAAGAAGGG \\
\hline SH3-L2 R & CTAGTGATGGTGATGATGATGACCACTACCTCCACCAGAACCTCCACCTCCACCAATCAAAGCAGAAACAGAAG \\
\hline L2-ADH2t F & GAACAAAAGTTGATCTCCGAAGAAGATTTGTAGGCGGATCTCTTATGTCTTTACGATTTA \\
\hline L1-SYN1 F & GGTGGAGGTTCTGGTGGAGGTAGTGGTAACTTGGTTGCCCAATTGGAAAAC \\
\hline SYN1-L2R & CTAGTGATGGTGATGATGATGACCACTACCTCCACCAGAACCTCCACCTTCTTCAATCTTCTTTCTTAAGTTGGCGATTT \\
\hline L1-SYN3 F & GGTGGAGGTTCTGGTGGAGGTAGTGGTAACGAAGTTACCACCTTGGAAAATGA \\
\hline SYN3-L3 R & CTAGTGATGGTGATGATGATGACCACTACCTCCACCAGAACCTCCACCTTTCTTATGGGCCAATCTGTTTCTCAA \\
\hline L1-GBD F & GGTGGAGGTTCTGGTGGAGGTAGTGGTAAGGCCGATATTGGTACTCCATCTAA \\
\hline GBD-L2 R & CTAGTGATGGTGATGATGATGACCACTACCTCCACCAGAACCTCCACCTGGGGCTTGTCTTCTCAATTCATTC \\
\hline L1-GHF & GGTGGAGGTTCTGGTGGAGGTAGTGGTGATGTCATGTGGGAATACAAGTGGG \\
\hline GHF-L2 & CTAGTGATGGTGATGATGATGACCACTACCTCCACCAGAACCTCCACCGGTGTACAAGTCGAAGTCAATTCTCTT \\
\hline TEF2p-L1 R & ACCACTACCTCCACCAGAACATTGTTTTTTAATTATAGTTCGTTGACCGTATATTCT \\
\hline L1-SH3lig F & GGTGGAGGTTCTGGTGGAGGTAGTGGTCCACCACCAGCTTTGCCAC \\
\hline SH3lig-L3 R & $\begin{array}{l}\text { CAAATCTTCTTCGGAGATCAACTTTTGTTCACCACTACCTCCACCAGAACCTCCACCTCTTCTTCTTTTTGGTGGCAAAGCTT } \\
\text { AG }\end{array}$ \\
\hline L2-Eno2t F & TTCTGGTGGAGGTAGTGGTCATCACCACCATCACCATTAGAGTGCTTTTAACTAAGAATTATTAGTCTTTTC \\
\hline L1-SYN2 F & GGTGGAGGTTCTGGTGGAGGTAGTGGTATTAACTATTTTACACCAGGCATATAGTGGTTTG \\
\hline SYN2-L2 R & $\begin{array}{l}\text { CTACAAATCTTCTTCGGAGATCAACTTTTGTTCACCACTACCTCCACCAGAACCTCCACCGATCTTTTTTGCATAACGTGCA } \\
\text { TCAAAGC }\end{array}$ \\
\hline L1-SYN4 F & GGTGGAGGTTCTGGTGGAGGTAGTGGTCAAAAGGTTGCCGAATTGAAGAATAGAG \\
\hline SYN4-L2 R & CTAGTGATGGTGATGATGATGACCACTACCTCCACCAGAACCTCCACCTTCAGCGACATCATTTTCCAATCTGG \\
\hline L1-GBDlig F & GGTGGAGGTTCTGGTGGAGGTAGTGGTTTGGTTGGTGCTTTGATGCACGTTA \\
\hline
\end{tabular}




\begin{tabular}{|l|l|}
\hline L3-GBDlig $\mathrm{R}$ & $\begin{array}{l}\text { CAAATCTTCTTCGGAGATCAACTTTTGTTCACCACTACCTCCACCAGAACCTCCACCATCTTCATCTTCGTCACCAGCTTGA } \\
\text { TAG }\end{array}$ \\
\hline L1-GHFlig F & GGTGGAGGTTCTGGTGGAGGTAGTGGTGAATTTGGTCCACCACCAGGTC \\
\hline L3-GHFlig R & $\begin{array}{l}\text { CTACAAATCTTCTTCGGAGATCAACTTTTGTTCACCACTACCTCCACCAGAACCTCCACCCAATCTTTGACCTGGTGGTGGA } \\
\text { C }\end{array}$ \\
\hline NVenus-ADH2t $\mathrm{F}$ & ctataactctcacaatgtttacatcactgctgacaaacaaTAGGCGGATCTCTTATGTCTTTACGATT \\
\hline NVenus-ADH2t $\mathrm{R}$ & GATAATGAAAACTATAAATCGTAAAGACATAAGAGATCCGCCTAttgtttgtcagcagtgatgtaaacattg \\
\hline CVenus-ENO2t $\mathrm{F}$ & tgctggtattacccatggtatggatgaattgtacaaataaTAGagtgcttttaactaagaattattagtctttctgc \\
\hline CVenus-ENO2t $\mathrm{R}$ & aataagcagaaaagactaataattcttagttaaaagcactCTAttatttgacaattcatccataccatgggta \\
\hline
\end{tabular}




\subsection{Integration of the $\operatorname{erg} 20^{\mathrm{K} 197 \mathrm{E}}$ mutation}

The chromosomal knock-in of the $\operatorname{erg} 20^{\mathrm{K} 197 \mathrm{E}}$ mutation was done by homologous recombination using an antibiotic marker-containing disruption cassette created by PCR, as described by Rothstein. $\operatorname{erg} 20^{\mathrm{K} 197 \mathrm{E}}$ was generated by overlap extension PCR to introduce the base pair change corresponding to K197E. The mutation was integrated as a $\mathrm{P}_{\text {TPII-erg20 }}{ }^{\mathrm{K} 197 \mathrm{E}}-\mathrm{T}_{\text {CYCl-loxP-kanMX-loxP }}$ cassette flanked by homology to the ERG20 chromosomal locus. Our attempts to introduce the K197E mutation into a haploid strain failed, possibly due to the combined stress of the transformation process and the metabolic burden of the mutation to a single copy of an essential gene. To decouple these effects, the cassette was transformed into a diploid strain and transformants were selected on YPD supplemented with $200 \mu \mathrm{g} / \mathrm{mL} \mathrm{G} 418$. The diploid transformants were sporulated and segregated to generate the haploid mutant strain. Stability of the integration was verified after several propagations and the erg20 $0^{\mathrm{K} 197 \mathrm{E}}$ locus was PCR amplified and sequence verified. This mutation was introduced into both double and quadruple auxotrophic yeast in the same manner.

\subsection{Analysis of Monoterpenoid Production in S. cerevisiae}

To measure monoterpenoid production, strains were seeded in triplicate to an $\mathrm{OD}_{600}$ of 0.05 in $40 \mathrm{~mL}$ of SC medium in $250 \mathrm{~mL}$ flasks and incubated at $30^{\circ} \mathrm{C}$ and $200 \mathrm{rpm}$ over a period of 4 days. For monoterpenoid analysis, $1-\mathrm{mL}$ culture samples were extracted into 0.25 volumes of ethyl acetate containing $10.7 \mathrm{mg} / \mathrm{L}$ of eugenol as internal standard. Extracts were analyzed by GC-EI-MS using an Agilent $6890 \mathrm{~N}$ GC system coupled to an Agilent 5875 mass selective detector. Three $\mu \mathrm{L}$ of extracts were injected in pulsed splitless mode at 50 PSI for 0.5 min with an injection temperature of $250^{\circ} \mathrm{C}$. Hydrogen was used as a carrier gas at a constant flow of $1 \mathrm{~mL} / \mathrm{min}$. A 10-min temperature gradient ranging from 80 to $300^{\circ} \mathrm{C}$ was used to separate analytes over a DB-5ms column $(25 \mathrm{~m} \times$ $0.20 \mathrm{~mm} \times 0.33 \mu \mathrm{m}$ film thickness). Analytes were identified and quantitated using linear 
calibration curves with authentic standards in concentrations ranging up to $100 \mathrm{mg} / \mathrm{L}$. Geraniol concentration was evaluated by scanning for three ions: 93, 123 and $154 \mathrm{~m} / z$. Nepetalactol was measured by following ions 135 and $168 \mathrm{~m} / \mathrm{z}$. Mevalonate was estimated by monitoring only $58 \mathrm{~m} / \mathrm{z}$. Linalool, citronellol, eugenol and 10hydroxygeraniol were measured using the Total Ion Chromatogram (TIC) scanning for $\mathrm{m} / \mathrm{z}$ values of $50-220$. For lack of authentic standards in the cases of 10hydroxycitronellol and 10-hydroxylinalool, concentrations were estimated using the 10hydroxygeraniol standard curves. Means are reported and errors are given as confidence interval $(\mathrm{p}<0.05)$.

\subsection{0-Hydroxygeraniol Oxidoreductase and Iridoid Synthase Assays}

Yeast cellular extracts were prepared in order to measure 10-hydroxygeraniol oxidoreductase and Iridiod synthase activity. To prepare the extracts, overnight cultures of the strain expressing $\mathrm{CrIS}, \mathrm{Cr} 10 \mathrm{HGO}, \mathrm{Cr} 10 \mathrm{HGO}-\mathrm{CrIS}$ or an empty vector control were used to inoculate $100 \mathrm{~mL}$ of $\mathrm{SC}$ medium to an $\mathrm{OD}_{600}$ of 0.05 . After 24 hours of growth, cells from triplicate $15-\mathrm{mL}$ volumes of each culture were harvested by centrifugation for $5 \mathrm{~min}$ at $3200 \times \mathrm{g}$. For the $\mathrm{CrIS}$ assays, cells were washed twice with $900 \mu \mathrm{L}$ of $150 \mathrm{mM}$ MOPS pH 7 and placed in a $2-\mathrm{mL}$ conical microtube with silicon Oring. Approximately $0.1 \mathrm{~mL}$ of glass beads were added to each tube and the cells were lysed by 3 x 30 seconds long cycles at $6000 \mathrm{rpm}$ in a Precellys homogenizer. The homogenizer was maintained at $4^{\circ} \mathrm{C}$ with 45 seconds between cycles. Immediately after lysis, $100 \mu \mathrm{L}$ of a stock solution containing $10 \mathrm{x}$ protease inhibitor $(1 \mathrm{tablet} / \mathrm{mL}$, Complete Mini EDTA free, Roche) and $5 \mathrm{mM}$ of both geraniol and NADPH were added to the lysates to achieve a final concentration of $500 \mu \mathrm{M}$. For $\mathrm{Cr} 10 \mathrm{HGO}, \mathrm{Cr} 10 \mathrm{HGO}-\mathrm{Cr} \mathrm{IS}$ assays, $50 \mathrm{mM}$ Tris $\mathrm{pH} 9$ was used for lysis and $100 \mu \mathrm{L}$ of a solution containing $5.45 \mathrm{mM}$ 10-hydroxygeraniol, $10 \mathrm{mM} \mathrm{NAD}^{+}$and 10x protease inhibitor (Complete Mini, EDTA free, Roche) was added to $900 \mu \mathrm{L}$ the lysates. This gave a final concentration of $545 \mu \mathrm{M}$ 10-hydroxygernaiol and $1 \mathrm{mM} \mathrm{NAD}^{+}$. $\mathrm{Cr} 10 \mathrm{HGO}$ catalyzes two successive oxidations, but 
at low $\mathrm{pH}$ can also act as a reductase and perform the reverse reactions [22]. Therefore, assays were run at $\mathrm{pH} 9$ to maximize the oxidative activity of $\mathrm{Cr} 10 \mathrm{HGO}$. All assay samples were incubated for 1 hour at $30^{\circ} \mathrm{C}$ then extracted with ethyl acetate and analyzed by GC-EI-MS as described above.

\subsection{Split Venus Protein-Protein Interaction Assay}

Protein fragments to be tested for interaction were fused 5 ' with either the N-terminal (amino acids 1-158) or C-terminal (amino acids 159-239) domains of the YFP variant Venus. The Venus-domain fusions were cloned into plasmids under the expression of a constitution promoter and complementary protein fragments were co-transformed into $S$. cerevisiae. If protein binding is successful, the Venus fragments should reassemble and the fluorescent signal will be reconstituted (Figure 8). Cells containing complementary Venus fragments were grown until mid logarithmic phase, where a 100 ul sample was collected, washed once in PBS, then re-suspended in $1 \mathrm{ml}$ PBS for analysis. Data was collected using a BD Accuri ${ }^{\mathrm{TM}} \mathrm{C} 6$ flow cytometer. Gates were set around a nonfluorescent control to standardize for forward (FSC) and side (SSC) light scattering. All fluorescence data was collected using an emission laser set at 488nm and a 530/30nm emission filter (FL1). 


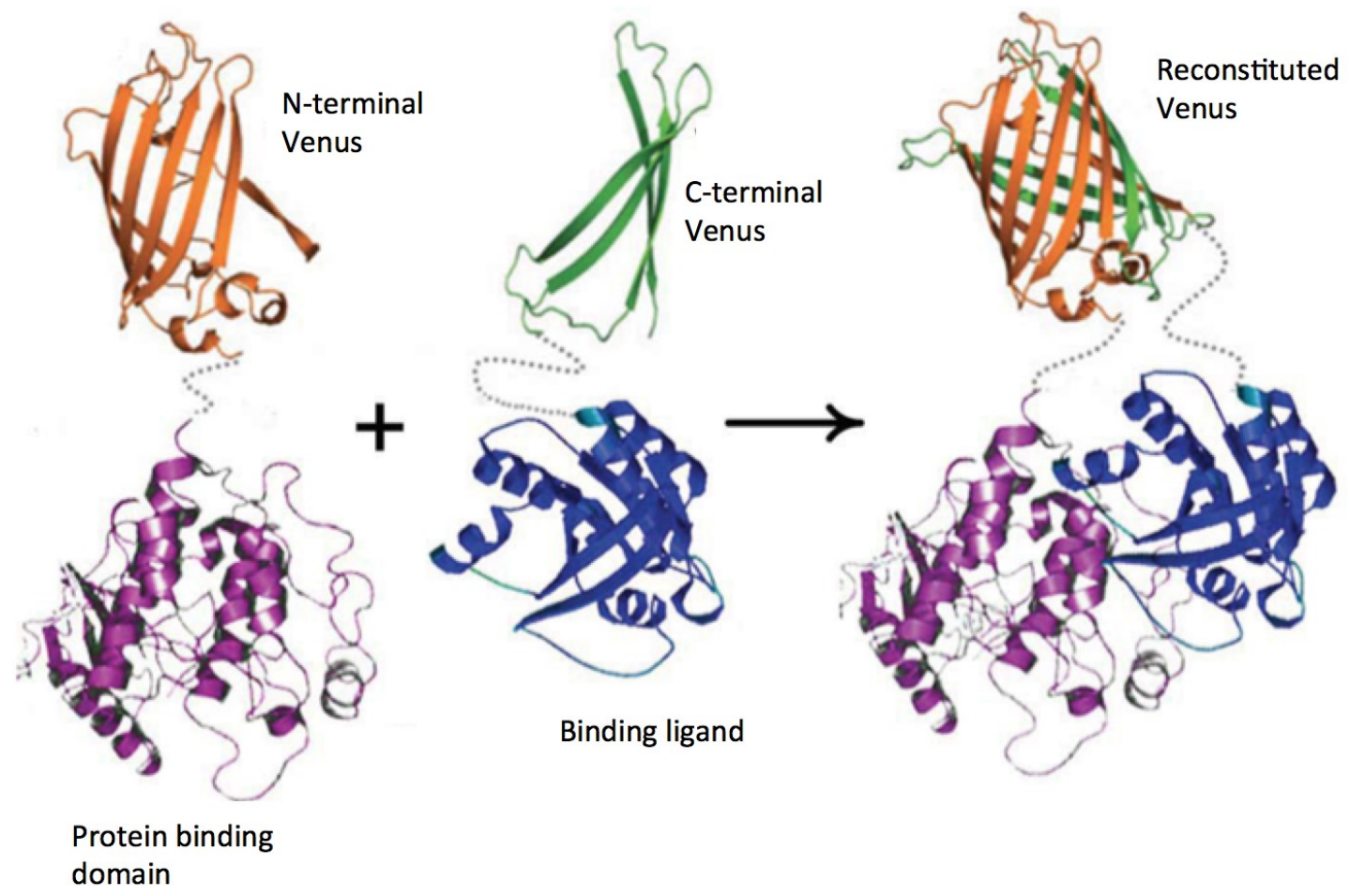

Figure 8: Split Venus protein-protein interaction assay. Protein binding partners are fused to the Nterminal and C-terminal fragments of a Venus fluorescent protein. If the proteins successfully bind, Venus is reconstituted and the fluorescent signal is restored. Adapted from [77].

\section{RESULTS}

\subsection{Geraniol synthesis in $S$. cerevisiae}

As a first step towards achieving 10-hydroxygeraniol synthesis in yeast, increased metabolic flux to GPP was engineered in a wild-type strain. As GPP is unstable and causes feedback inhibition to the upstream gene mevalonate kinase [48], geraniol synthesis was used as our read-out. Expression of the O. basilicum geraniol synthase $(O b G E S)$ [78] was used to convert GPP to geraniol in yeast. Additionally, flux through 
the mevalonate pathway was increased by the recombinant expression genes $E R G 13$, tHMGR, ERG8 and ERG12 (Figure 6). Expression of these four genes was tested from either an episomal $2 \mu(\mathrm{MevH})$ or a centromeric (MevL) plasmid, and strains were tested for geraniol productivity as well as mevalonate to determine the extent of feedback inhibition.

The highest specific production of geraniol observed was from a strain that had the mevalonate pathway overexpressed from a low-copy centromeric plasmid, along with the $\operatorname{erg} 20^{\mathrm{K} 197 \mathrm{E}}$ chromosomal mutation and the geraniol-producing enzymes $\mathrm{AgGPPS} 2$ and ObGES (Figure 9). In the erg $20^{\mathrm{K} 197 \mathrm{E}}$ background, higher expression of the mevalonate pathway did not result in increased concentration of geraniol as compared to low-copy expression. In the background of $\operatorname{erg} 20^{\mathrm{K} 197 \mathrm{E}}$ and in the presence of $A g$ GPPS2 and ObGES, still low-copy expression of the mevalonate pathway generated more geraniol than high-copy expression (Figure 9). This could be due to flux imbalance as the mevalonate pathway intermediate HMG-CoA is known to be toxic [47]. In addition, mevalonate was found to accumulate to a higher level in the absence of AgGPPS2ObGES expression (Figure 7), likely caused by the feedback inhibition of mevalonate kinase $S c$ ERG12 by GPP/FPP [79] and further supporting the importance of pulling on the intracellular GPP pool (Figure 9). Although introducing the $\operatorname{erg} 20^{\mathrm{K} 197 \mathrm{E}}$ mutation resulted in improved geraniol production levels, a $\sim 66 \%$ decrease in cell density over wild-type was observed, which is consistent with previous results [80]. This could be due to geraniol toxicity [81] or reduced intracellular FPP pools ultimately limiting ergosterol biosynthesis [82]. 


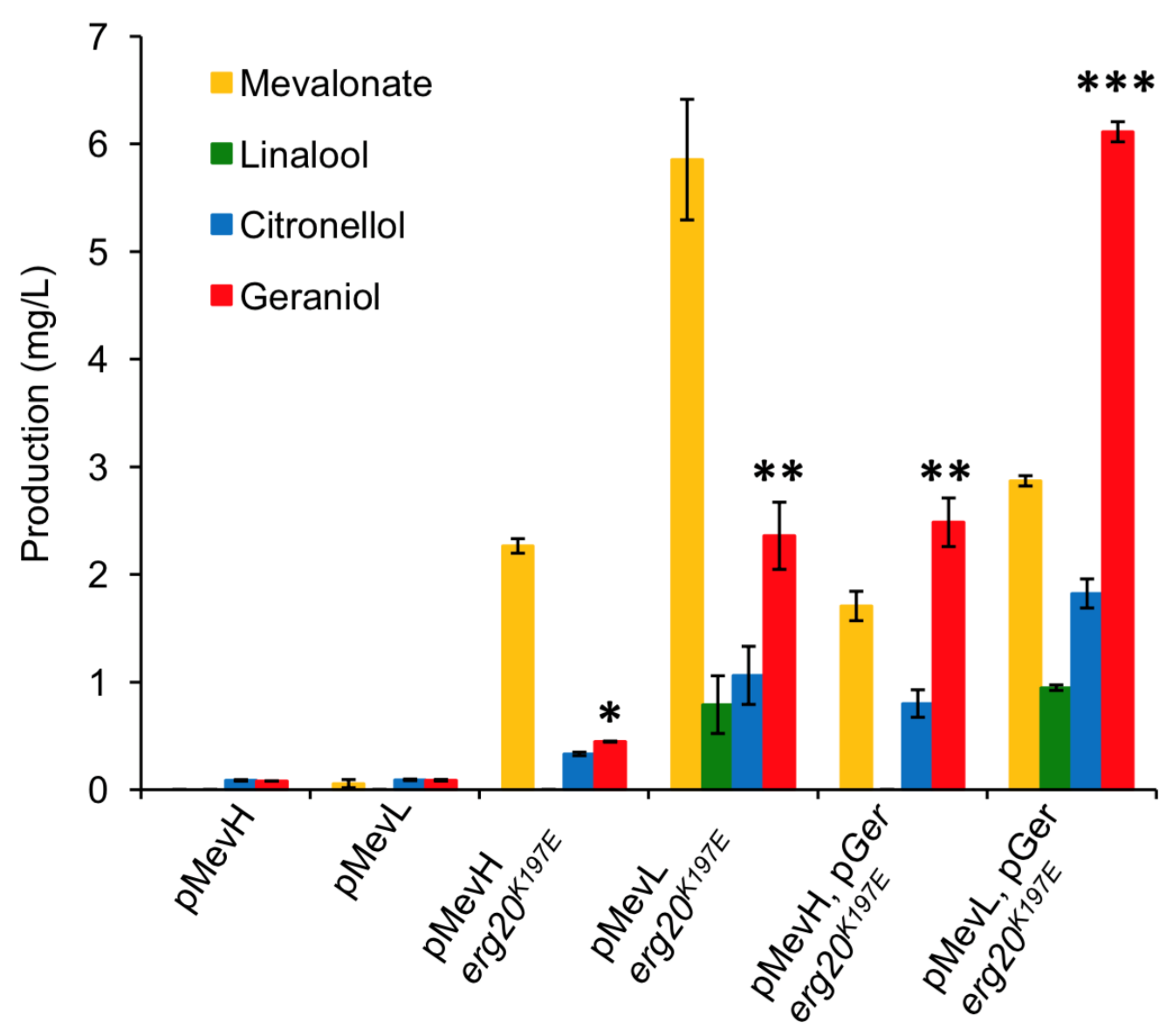

Figure 9: Specific production of the monoterpenoids and mevalonate by engineered S.cerevisiae strains. Mevalonate pathway genes ERG8, ERG12, ERG13 and tHMGR were overexpressed from a high-copy (pMevH) or low-copy (pMevL) plasmid. The GES, GPPS2 and CPR genes were expressed together from a high-copy plasmid (pGer). Wildtype $E R G 20$ background strain was compared with an $\operatorname{erg} 20^{K 197 E}$ mutant strain favoring production of GPP over FPP. Asterisks indicate statistically significant $(\mathrm{p}<0.05)$ improvement in geraniol production, as determined by Student's paired $t$-test with twotailed distribution. Annotations of $*, * *$ and $* * *$ represent statistical differences to the control MevH or MevL strains. Error bars represent the $95 \%$ confidence interval from triplicate cultures inoculated from a single colony. Note: This experiment was performed by Nick Gold and Philippe Bauchart. 
The production of linalool and citronellol was also measured. Linalool was only observed from strains expressing the mevalonate pathway from a low-copy plasmid whereas citronellol production followed geraniol, as expected. Based on these results, the geraniol-producing strain with the highest yield (pMevL, pGer, erg20 ${ }^{\mathrm{K} 197 \mathrm{E}}$ ) was used as the starting strain to further extend the Iridiod pathway.

\subsection{0-Hydroxygeraniol Synthesis in S. cerevisiae}

Using our highest geraniol-producing strain, we aimed to show activity for the next step in the Iridiod pathway, production of 10-hydroxygeraniol. This reaction is catalyzed by the cytochrome $\mathrm{P} 450$ monooxygenase $\mathrm{Cr} \mathrm{G} 10 \mathrm{H}$ and requires the accessory reductase $\mathrm{CrCPR}$ [59]. To test the functionality of the monooxygenase in yeast, the genes encoding the two enzymes were added to the AgGPPS2-ObGES expressing plasmid and both the geraniol and 10-hydroxygeraniol-producing strains were tested in parallel. Expression of the $\mathrm{CrG10 \textrm {H }}$ in our geraniol-producing strain resulted in a peak of $5.3 \mathrm{mg} / \mathrm{L}$ of 10 hydroxygeraniol (Figure 10). In addition, compounds inferred to be 10hydroxycitronellol and 10-hydroxylinalool by MS analysis were also observed in the $\mathrm{Cr}$ G10H-expressing strain. One of the peaks (Figure 10, Peak 7) was identified as having $62 \%$ probability match for 10-hydroxylinalool (NIST08 MS library), while the identity of 10-hydroxycitronellol (Figure 10, Peak 9) was inferred from (i) the presence of the 154 $\mathrm{m} / \mathrm{z}$ daughter ion $\left(\mathrm{M}^{+} 172 \mathrm{~m} / \mathrm{z}\right.$ with loss of water as $\left.-18 \mathrm{~m} / \mathrm{z}\right)$ and a match to previously reported MS spectra [83]; (ii) its retention time relative to 10-hydroxylinalool and 10hydroxygeraniol and how its elution time relative to citronellol concords with those of geraniol and linalool and their corresponding hydroxylated products (Figure 10A) and; (iii) its signal profile over time and how it tracks with that of 10-hydroxygeraniol in a similar fashion to the way that citronellol tracks with geraniol (Figure 10C). The presence of both the proposed 10-hydroxylinalool and 10-hydroxycitronellol in the $\mathrm{Cr} \mathrm{G} 10 \mathrm{H}$ expressing strain suggests that this enzyme is promiscuous and can hydroxylate a range of monoterpenes. Phenylethanol and tyrosol were detected at comparable levels in all strains including wild-type controls with pMevL or pMevH (data not shown). These fusel 
alcohols are standard yeast metabolites with no relation to the MIA pathway[83,84]
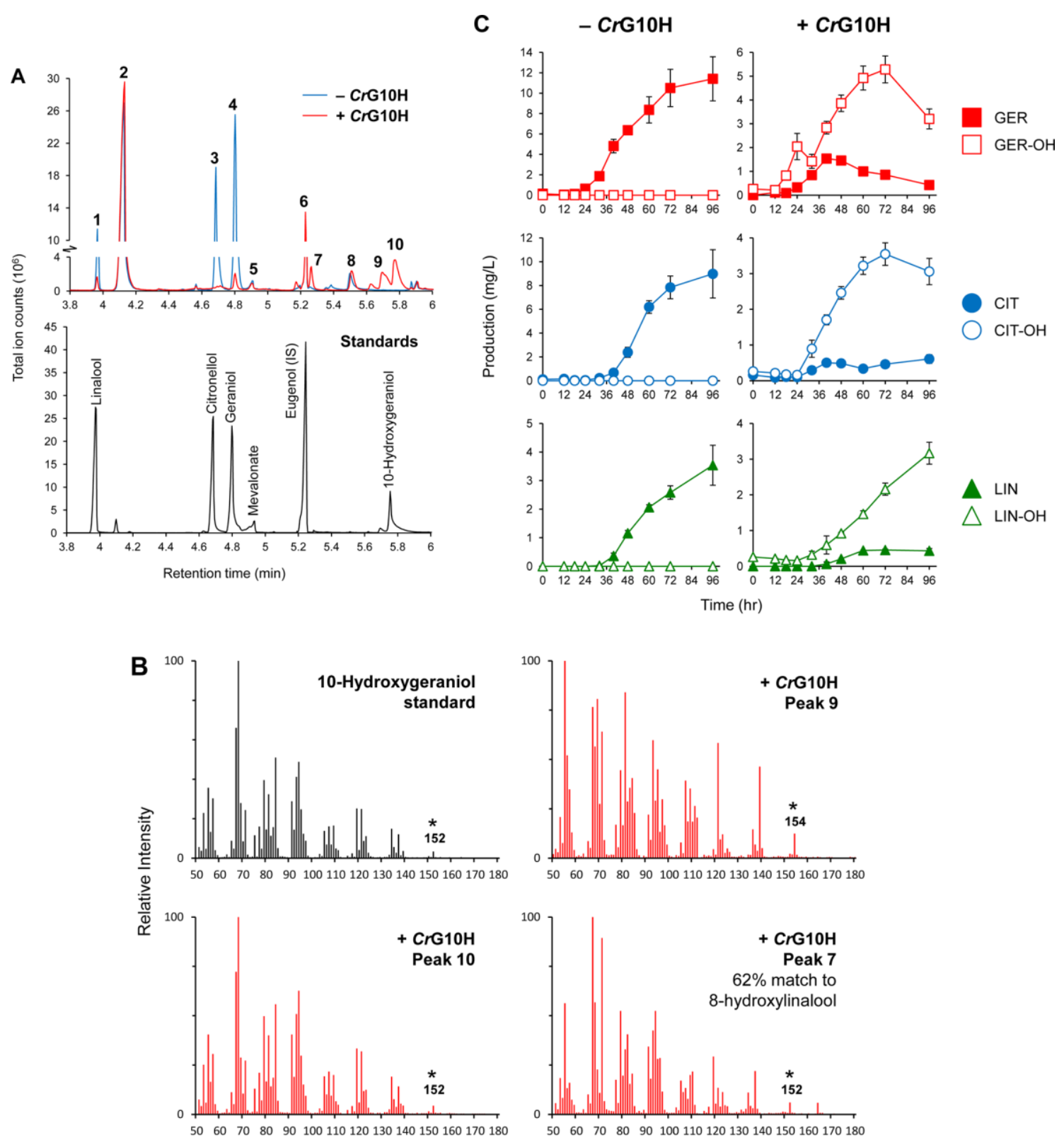

Mass-to-charge ratio

Figure 10: Production of monoterpenoids from geraniol-producing strains without (-CrG10H) and with $(+\mathrm{CrG10H})$ heterologous expression of C.roseus geraniol 10hydroxylase A. Representative total ion chromatographic profiles of monoterpenoids extracted from culture broths. Peak identifications using analytical standards are as follows: 1, linalool; 3, citronellol; 4, geraniol; 5, mevalonate; 6, eugenol (internal 
standard); 10, 10-hydroxygeraniol. Peak 7 was identified by NIST08 MS Search 7.0 as a $62 \%$ probability match for 10 -hydroxylinalool. Unknown peak 9 is proposed to be 10 hydroxycitronellol. Peaks 2 and 8 correspond to phenylethanol and tyrosol, respectively, which were found to accumulate in all samples, including wild-type controls with $\mathrm{pMevL}$ or pMevH (not shown). These fusel alcohols are standard yeast metabolites with no relation to the MIA pathway. B. Representative EI-MS spectra of 10-hydroxygeraniol (peak 10) and peaks proposed to correspond to 10-hydroxycitronellol (peak 9) and 10hydroxylinalool (peak 7). Asterisks identify the $\mathrm{M}^{+}-18$ peak. C. Monoterpenoid production profiles from geraniol-producing strains without $(-\mathrm{Cr} \mathrm{G} 10 \mathrm{H})$ or with $(+\mathrm{Cr} \mathrm{G} 10 \mathrm{H})$ co-expression of the geraniol 10-hydroxylase. Error bars represent the $95 \%$ confidence interval from triplicate cultures inoculated from a single colony. Note: This experiment performed by Nick Gold and Philippe Bauchart.

\subsection{The Iridoid Synthase $\boldsymbol{C r I S}$ is a Promiscuous Reductase}

After successful synthesis of 10-hydroxygeraniol in yeast, we sought to extend the pathway to 10-oxogeranial by the co-expression of the C. roseus 10-hydroxygeraniol oxidoreductase $(\mathrm{Cr} 10 \mathrm{HGO})$ in our 10-hydroxygeraniol-producing strain. Although depletion of 10-hydroxygeraniol was observed indicating that the $\mathrm{CrHGO}$ was active in this strain, we were unable to detect 10-oxogeranial or 10-hydroxygeranial. Difficulty in measuring 10-oxogeraniol was probably due to its highly reactive aldehyde group. To get around this problem, we opted to extend the Iridiod pathway with the addition of the $C$. roseus Iridiod synthase ( $\mathrm{Cr} \mathrm{IS})$ to produce nepetalactol. Surprisingly, nepetalactol, geraniol or 10-hydroxygeraniol was not detected but instead a striking $200 \mathrm{mg} / \mathrm{L}$ of citronellol and $5.6 \mathrm{mg} / \mathrm{L}$ of proposed 10-hydroxycitronellol were observed (Figure 11A). Based on these results, we speculated that $\mathrm{CrIS}$, which is an NADPH-dependent reductive cyclase, might be capable of reducing geraniol and 10-hydroxygeraniol without cyclizing them. In addition, the CrIS is known to be promiscuous for a number of monoterpenes [86]. To test this hypothesis lysates from yeast expressing the $\mathrm{CrIS}$ alone 
were incubated with geraniol and NADPH. As expected, lysates of cells expressing the CrIS produced 10.6 times more citronellol than the negative control when incubated in the presence of geraniol and 3.1 times more proposed 10-hydroxycitronellol when incubated in the presence of 10-hydroxygeraniol (Figure 11B and 11C), further supporting the identity of unknown peak 7 (Figure 10) as 10-hydroxycitronellol. The presence of citronellol and 10-hydroxycitronellol in the control lysates is likely due to the endogenous yeast proteins Oye 2 and Oye3, which are reductases that are known to be active on geraniol [87]. By comparing these enzyme assays to the in vivo data (Figure 11A) we concluded that $\mathrm{CrIS}$ outcompetes $\mathrm{CrG10H}$ for geraniol and that $\mathrm{CrG} 10 \mathrm{H}$ is equally poor at converting citronellol to 10-hydroxycitronellol as OYE2/OYE3 is at converting 10-hydroxygeraniol to 10-hydroxycitronellol. Furthermore, our results suggest that $\mathrm{Cr} 10 \mathrm{HGO}$ is active on 10-hydroxycitronellol since its concentration went down only in our $\mathrm{Cr} 10 \mathrm{HGO}$-expressing strain (Figure 10A and Figure 11A). These experiments provide direct evidence that $\mathrm{CrIS}$ is capable of reducing geraniol to citronellol and 10hydroxygeraniol to 10-hydroxycitronellol thereby diverting carbon away from the desired products, 10-oxogeranial and nepetalactol. 

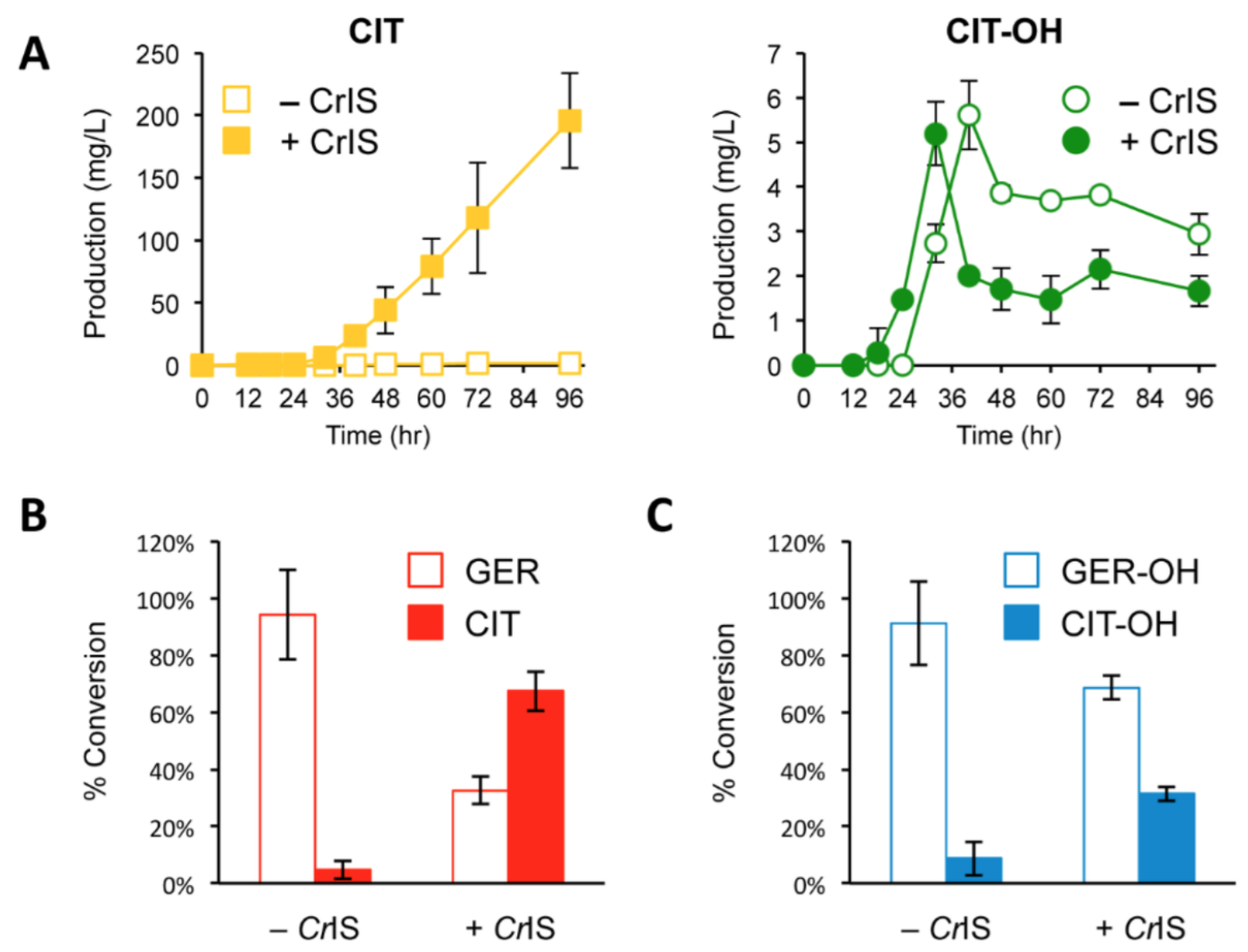

Figure 11: Evidence of promiscuity of CrIS A. Citronellol (CIT) and 10hydroxycitronellol (CIT-OH) production by 10-hydroxygeraniol-producing strain coexpressing either $\mathrm{Cr} 10 \mathrm{HGO}$ (-CrIS) or Cr10HGO-CrIS (+CrIS). B. Percentage of geraniol (GER) converted to citronellol (CIT) when incubated with lysates from wildtype yeast (-CrIS, empty vector) or lysates from yeast cells expressing $\mathrm{Cr}$ IS (+CrIS) $\mathbf{C}$. Percentage of 10-hydroxygeraniol (GER-OH) converted to 10-hydroxycitronellol (proposed; CIT-OH) when incubated with lysates from wild-type yeast (-CrIS, empty vector) or lysates from yeast cells expressing $\mathrm{CrIS}(+\mathrm{Cr} \mathrm{IS})$. Error bars represent the $95 \%$ confidence interval from triplicate cultures inoculated from a single colony. 


\subsection{In vitro enzyme activity of $\mathrm{Cr}$ 10HGO-CrIS enzyme pair}

In order to demonstrate that the $C r 10 \mathrm{HGO}-\mathrm{CrIS}$ enzyme pair is active in $S$. cerevisiae, we tested lysates of cells expressing both enzymes for the production of nepetalactol using 10-hydroxygeraniol as a substrate. Since CrIS catalyzes an irreversible reaction, nepetalactol was expected to accumulate even with a low turnover rate. In addition, this strategy avoided the issue caused by $C r I S$ activity on geraniol. Lysates expressing both $\mathrm{Cr} 10 \mathrm{HGO}$ and $\mathrm{CrIS}$ did indeed produce nepetalactol from 10-hydroxygeraniol whereas control lysates from cells only expressing the $\mathrm{Cr} 10 \mathrm{HGO}$ did not (Figure 12). These results confirm the functionality of these enzymes in $S$. cerevisiae as well as their concerted activity in the synthesis of nepetalactol [60] and further support the conclusion that the absence of in vivo nepetalactol synthesis from our 10-hydroxygeraniol-producing strain is likely the result of CrIS activity on the upstream intermediate geraniol. 
A

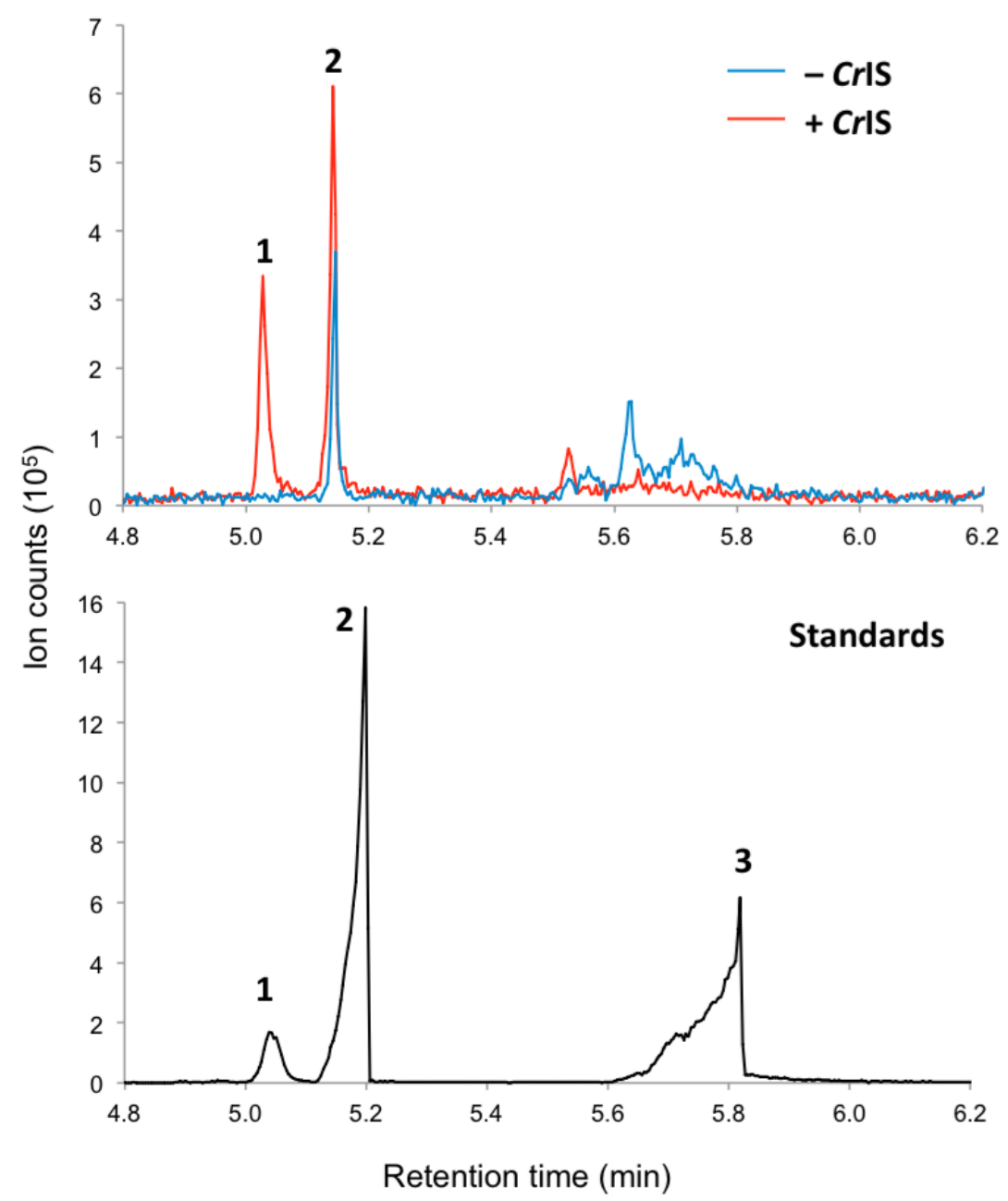

B
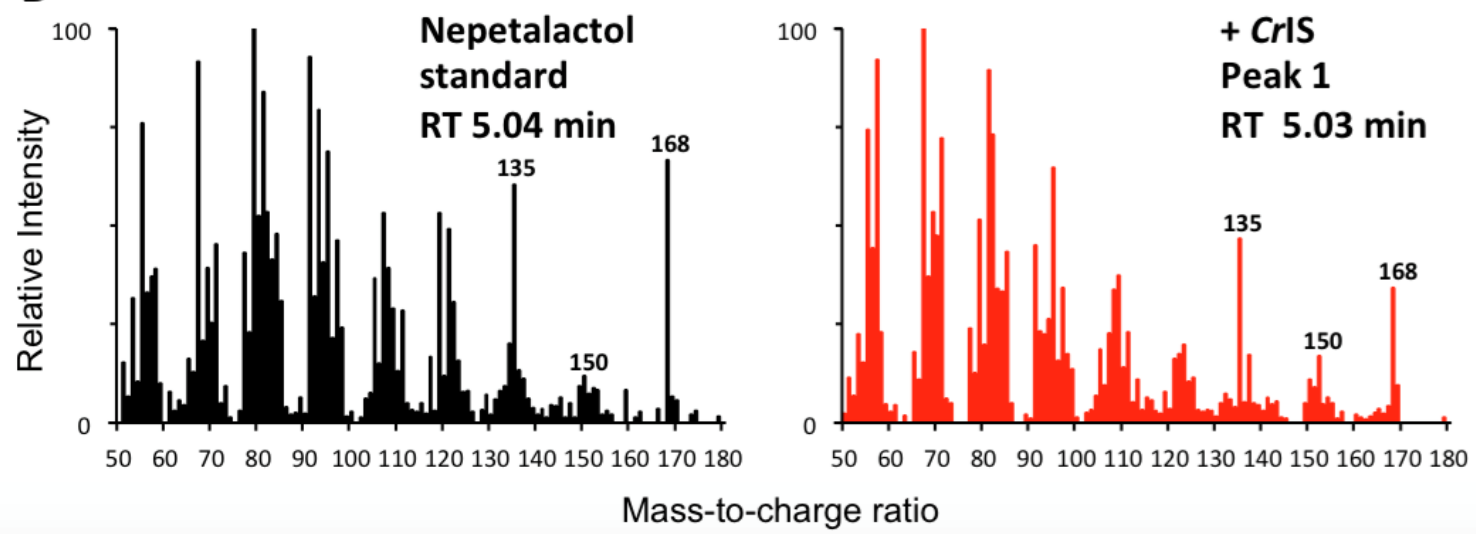

Figure 12: 10-hydroxygeraniol oxidase and iridodial synthase enzyme assays A. Selected ion monitoring (135 and $168 \mathrm{~m} / \mathrm{z}$ ) chromatograms for assays with 10hydroxygeraniol and $\mathrm{NAD}^{+}$using cell lysates for yeast strains expressing only $\mathrm{Cr} 10 \mathrm{HGO}$ 
(-CrIS) or both Cr10HGO and CrIS (+CrIS). Peaks were identified using analytical standards are as follows: 1, nepetalactol; 2, eugenol; 3, 10-hydroxygeraniol. B. MS spectra of peak 1 from +CrIS lysate compared with authentic nepetalactol standard.

\subsection{In vivo Characterization of Protein-Protein Binding Domains.}

Protein scaffolds were investigated as a potential method of improving metabolic flux within the MIA pathway. As a first step, 5 protein-protein binding domains were selected for testing based on their size, binding affinity and specificity (SYNZIP1, SYNZIP3, GBD, SH3 and GHF). These were functionally tested in yeast using a split Venus protein-protein interaction assay, where The $\mathrm{N}$ and $\mathrm{C}$ terminal fragments of a split Venus protein were fused to each protein binding domain and its cognate ligand. Successful binding in vivo was expected to reconstitute a fluorescent signal. A full version of the Venus protein, as well as Venus fragments lacking protein fusions ( $\mathrm{NV}_{\text {stop }}$ and $\left.\mathrm{CV}_{\text {stop }}\right)$ were used as a control. The results indicate that all domains successfully bound in vivo (Figure 13), as all domains fluoresced significantly more than associated control. The best domains were SYNZIP1/2 and SYNZIP3/4, which is unsurprising as these have been synthetically designed for high binding affinities [71]. We used this data to select SYNZIP3/4, GBD and SH3 for use in future protein scaffolding experiments. SYNZIP1/2 was excluded due to its documented cross-reactivity with SYNZIP3/4 [71], and GBD and SH3 were selected due to their well documented use in previous scaffolding experiments $[67,88]$. 


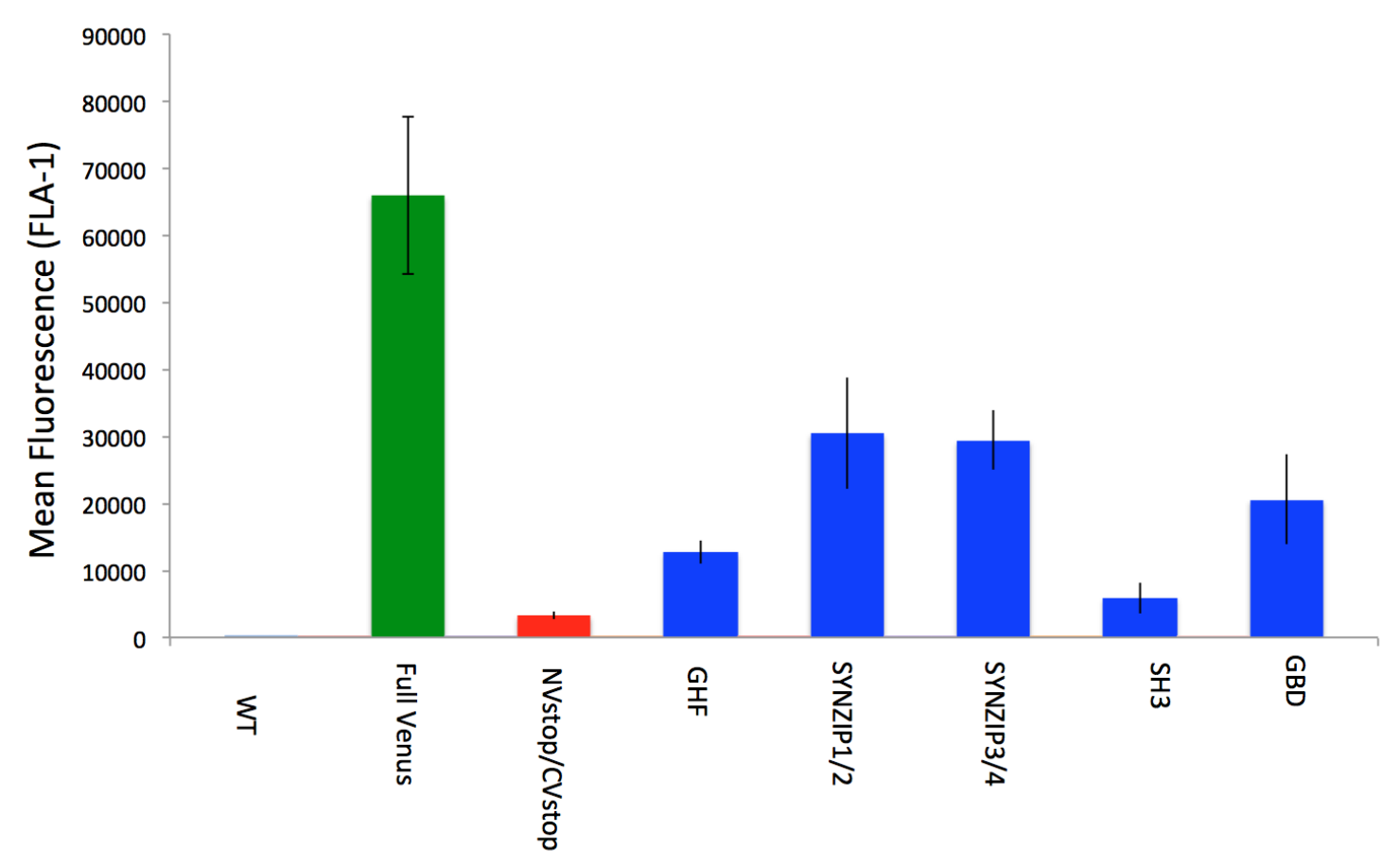

Figure 13: Split Venus protein-protein interaction assay. Five domains (SH3, GBD, SYNZIP1/2, SYZIP3/4 as well as GHF) were tested. A fully reconstituted Venus protein (green) as well as Venus fragments expressed without 3' protein fusions (red) were used as controls.

\subsection{Enhanced Production of MIA Metabolites using Protein Scaffolds.}

After validating that protein-protein binding occurred effectively in vivo, we sought to demonstrate the functional co-localization of MIA enzymes to a protein scaffold. The logical choice for this would have been the three steps covering the conversion of geraniol to iridodial that are vulnerable to attack by IS. At the time we were designing these experiments however, we were unaware of this activity and chose a different set of enzymes to scaffold. ERG20 ${ }^{\mathrm{K} 197 \mathrm{E}}$, GES, and old yellow enzyme 2 (OYE2) were used as candidates, which together catalyze the formation of citronellol from GPP (Figure 14). As GPP causes heavy feedback inhibition to mevalonate kinase $\left(\mathrm{K}_{\mathrm{i}}=0.25 \mu \mathrm{M}\right)$ its quick consumption through scaffolding should improve overall flux through the pathway. Furthermore, GPP is highly vulnerable intermediate and can be converted to both FPP 
and linalool. By using citronellol as an end product, we hoped to avoid the toxicity associated with high geraniol producing strains.

A.
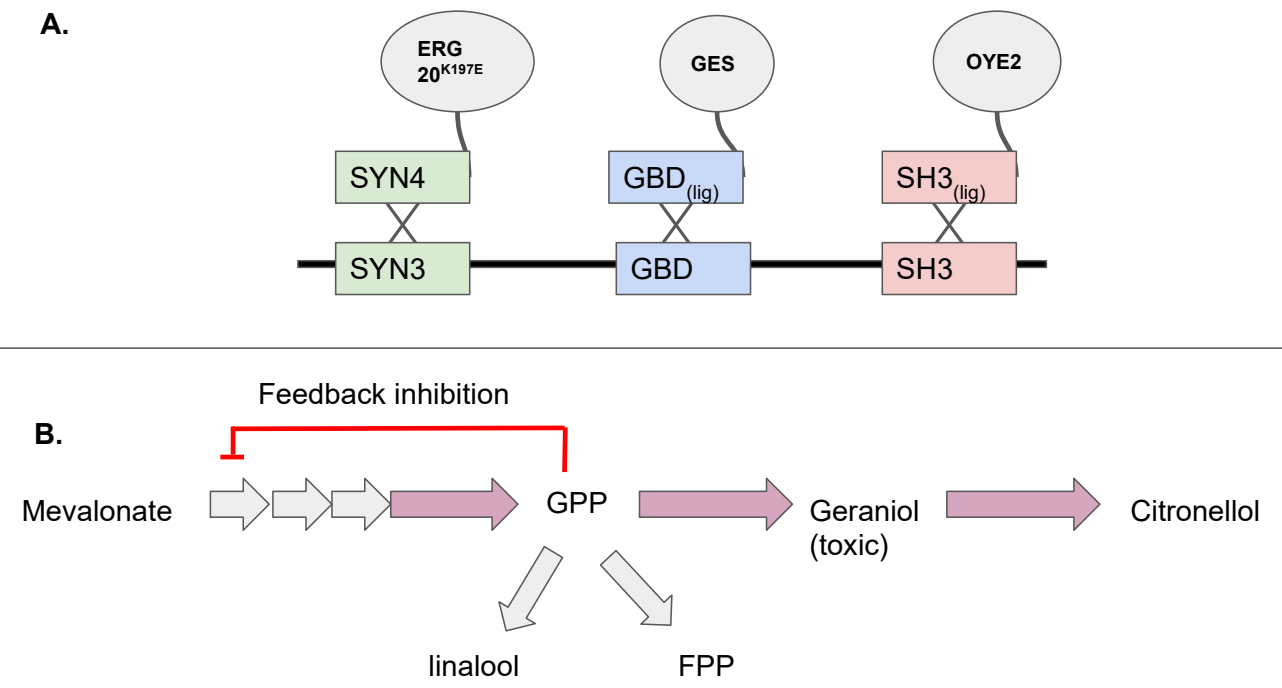

Figure 14: Depiction of citronellol based scaffolding experiments. A. The enzymes ERG20 ${ }^{\mathrm{K} 197 \mathrm{E}}$, GES and OYE2 are shown fused to their respective binding ligands, which are recruited to the scaffold B. Overview of the portion of the pathway being scaffolded, scaffolded enzymes are represented as purple arrows. 
Functional tests were performed by fusing the binding ligands SYN4, GBD (ligand) and $\mathrm{SH}_{\text {(ligand) }}$ to ERG ${ }^{\mathrm{K} 197 \mathrm{E}}$, GES, and OYE2 respectively. ERG20 ${ }^{\mathrm{K} 197 \mathrm{E}}$-SYN4 and GES$\mathrm{GBD}_{\text {(ligand) }}$ were expressed on PGREG504(trp) vector and OYE2-SH3 (ligand) was expressed on a pGREG506(ura) vector. Four variants of protein scaffold were built with architecture consisting of SYN3-(GBD) $)_{x}$-SH3 on a PGREG503(his) vector, where $\mathrm{x}=1-3$. Varying the number of GBD domains controls how many copies of GES are recruited to the scaffold, and should impact the rate at which GPP is metabolized. Domains were linked together by using a flexible glycine-serine linker with the amino acid sequence GGGSGGGSG, as used in other works [67] Each scaffold, along with the ERG-SYN4GES-GBD (ligand) $_{\text {and OYE2-SH3 }}$ (ligand) plasmids were transformed into an ERG20 K197E quadruple auxotroph expressing the pMevL plasmid. Strains were grown in triplicate and analyzed by GC-MS every 12 hours for the production of citronellol, geraniol, mevalonate and linalool (figure 15). 

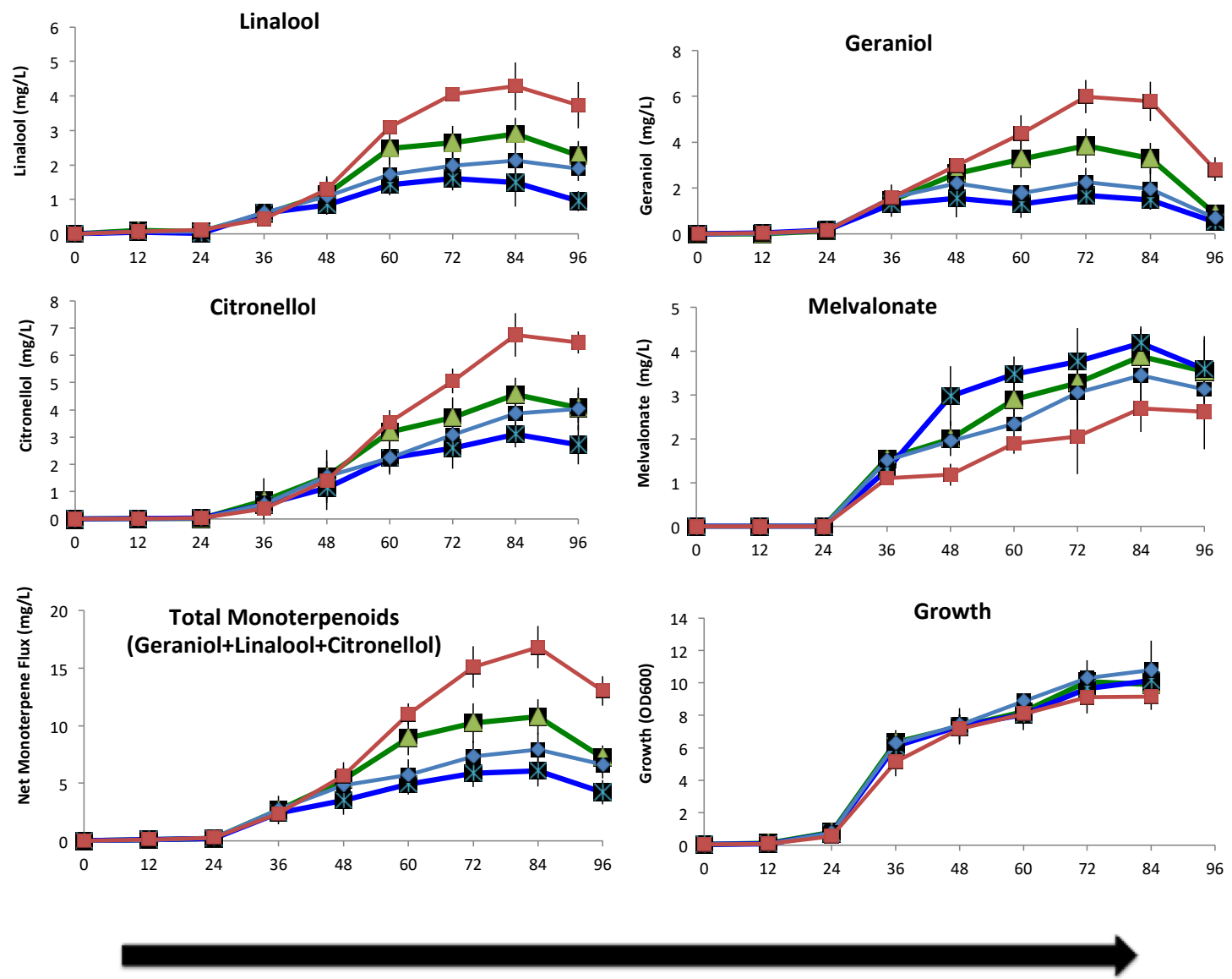

Time (Hours)

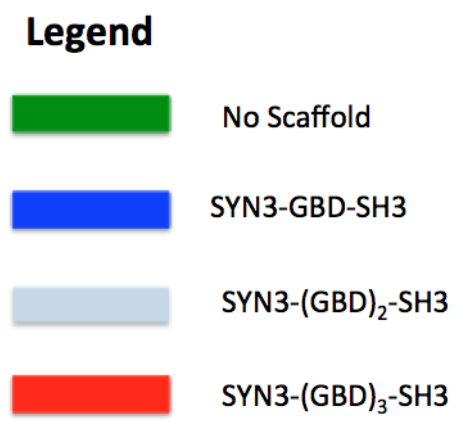

Figure 15: Production of monoterpenes and mevalonate in the presence of different scaffolds. Specific titers of geraniol, citronellol, mevalonate and linalool are shown for scaffolds possessing 1 (blue), 2(black) and 3(red) GBD domains as well as for the unscaffolded control (green). 
The results of this experiment indicated that monoterpene production was highly dependent on scaffold architecture. In general, scaffolds with more GBD domains tended to be the most effective. The lowest citronellol producer was the SYN3-(GBD) 1 -SH3 scaffold at $2.72 \mathrm{mg} / \mathrm{L}$. The variant with two SYN3-(GBD) $2-\mathrm{SH} 3$ was slightly better, producing $4.03 \mathrm{mg} / \mathrm{L}$ citronellol, though this was still slightly less than the un-scaffolded control $(4.07 \mathrm{mg} / \mathrm{L})$. This suggests that association with the protein scaffold may reduce the processivity of some enzymes. The best strain was with the scaffold possessing three GBD domains, which produced $6.46 \mathrm{mg} / \mathrm{L}$ citronellol, a $57.8 \%$ increase relative to the control. Production of all monoterpenes was also higher in this strain vs. the control (57.8\% increase for citronellol, $75.6 \%$ increase for geraniol, $44.7 \%$ increase for linalool). The increase in linalool was unexpected as higher producing geraniol/citronellol strains would be expected to have less GPP available to form linalool. Mevalonate levels were generally inversely proportional to the total monoterpene content (geraniol+linalool+cintrollol), with the best producer registering $21.86 \%$ less mevalonate the control, and 39.6\% less than the lowest (monoterpene) producer. This was expected as reduced mevalonate indicates alleviation of feedback inhibition, and should be associated with higher monoterpene content. No significant growth differences were observed between any of the strains.

\section{DISCUSSION}

\subsection{Improvement of Monoterpene Production}

With the goal of developing a platform for strictosidine biosynthesis, we initially aimed to engineer geraniol production in S. cerevisiae. Our strategy consisted of replacing the wild-type $E R G 20$ with the $\operatorname{erg} 20^{K 197 E}$ mutant allele combined with over-expression of the rate-limiting steps of the mevalonate pathway as well as GPP and geraniol synthases. In 
previous reports, strains harboring the $\operatorname{erg} 20^{K 197 E}$ mutation showed reduced FPP synthase activity, and favored monoterpenoid production with strains producing up to $0.455 \mathrm{mg} / \mathrm{L}$ geraniol [52]. The introduction of a plant geraniol synthase was also demonstrated to improve yields of monoterpenoids. For example, expression of the $O$. basilicum GES in an $\operatorname{erg} 20^{\mathrm{K} 197 \mathrm{E}}$ background resulted in the production of a 10 -fold higher monoterpenoid titer of $5 \mathrm{mg} / \mathrm{L}[79,87]$ Lastly, overexpression of enzymes from the endogenous mevalonate can give further improvement to geraniol titers. Our final engineered strain produced $11.4 \mathrm{mg} / \mathrm{L}$, a titer that is $\sim 3$ fold lower than previously reported for a similarly engineered geraniol-producing yeast strain. The difference between these values could be due to their over-expression of IDII and MAFI and the fact that a different variant of the $\operatorname{erg} 20^{K}$ mutant was used $\left(\operatorname{erg} 20^{K 197 G}\right.$ instead of $\operatorname{erg} 20^{K 197 E)}$ which may have lead to higher titers at the cost of lower cell growth [80]. Aside from geraniol, we also observed the related monoterpenoids linalool and citronellol. In S. cerevisiae, the old yellow enzymes OYE2 and OYE3 are thought to be responsible for non-specific reduction of geraniol to citronellol [57]. In our top geraniol-producing strain the ratio of citronellol to geraniol was 0.3 , pointing to the fact that this reaction may account for an important drain on the carbon in the pathway unless it is deleted from the strain, as previously shown [66]. The other major monoterpenoid, limonene, is a direct dephosphorylation product of GPP and was only observed in the strains pMevL, erg2 $0^{K 197 E}$ and pMevL, pGer, erg $20^{K 197 E}$ where their ratio of linalool to geraniol were 0.28 and 0.15 , respectively (Figure 9). Mevalonate was also measured since it is known to accumulate due to the inhibition of mevalonate kinase by GPP [48] making it a potential indicator of intracellular GPP pools. We observed that our strain engineered to stop at the intermediate GPP (pMevL, erg20 ${ }^{K 197 E}$ ) also produced the highest mevalonate titer and that mevalonate accumulation could be reduced, but not eliminated, by the additional expression of GES (pMevL, pGer, erg20 ${ }^{K 197 E}$ ) (Figure 9). This is expected as GES consumes GPP but also points to the fact that the conversion of GPP to geraniol is likely limiting flux in these strains.

\subsection{G10H expression and activity}


The geraniol-producing strain we engineered was used as a starting point for further extension of the Iridiod pathway. We first showed that the additional expression of the $C$. roseus geraniol 10-hydroxylase to this strain resulted in the accumulation of $5.3 \mathrm{mg} / \mathrm{L}$ of 10-hydroxygeraniol, a $46.3 \%$ turnover of geraniol. In addition, we observed two additional hydroxylated monoterpenoids and postulated that they were a product of $\mathrm{Cr} \mathrm{G} 10 \mathrm{H}$. $\mathrm{Cr} \mathrm{G} 10 \mathrm{H}$ activity has been reported on both linalool and citronellol therefore this is extremely probable. However, it is possible that some of the 10-hydroxycitronellol observed in this strain is a product of OYE2/OYE3 acting on 10-hydroxygeraniol.

\subsection{Impact of Iridiod synthase on Metabolite Distribution}

Upon further extension of the pathway by co-expression of 10-hydroxygeraniol oxidoreductase and Iridiod synthase, we expected to observe iridodial produced from 10hydroxygeraniol. However, only large amounts of citronellol and some putative 10hydroxycitronellol with no other monoterpenoids were detected. Since this phenomenon was not observed with the expression of 10HGO alone, we hypothesized that the Iridiod synthase might be responsible by reducing geraniol to citronellol. The Iridiod synthase is a reductive cyclase and although citronellol is not a cyclic product, we theorized that these two functions could be decoupled with reduction occurring independently of cyclization. We confirmed this activity in vitro by incubating IS-expressing yeast lysates with geraniol (Figure 11). A similar result was observed using 10-hydroxygeraniol although IS seems to have lower activity on this substrate. This observation is consistent with previous data, which has shown that IS is highly promiscuous and is active on at least five other monoterpenoids [86]. Most tellingly, IS retains $90 \%$ of its activity on citral - the aldehyde analog of geraniol [86]. It is therefore not surprising that IS would also have strong activity on geraniol itself. The observation has serious implications for the reconstitution of the Iridiod pathway in yeast.

In spite of the significant hurdles caused by side product reactions at several steps in the pathway, Brown et al. were recently successful at producing trace amounts of 
strictosidine in yeast from glucose [66]. By supplementing cultures with intermediates at progressively lower steps in the pathway, they identified geraniol as the failure point as supplementing this intermediate to the cells did not produce strictosidine. This bottleneck was solved by the expression of additional copies of $\mathrm{G} 10 \mathrm{H}$, which lead to the production of small amounts of strictosidine [66]. In light of our results, we postulate that overexpression of the $\mathrm{G} 10 \mathrm{H}$ by Brown et al. resulted in geraniol hydroxylation reaction outcompeting reduction by IS thereby channeling flux towards nepetalactol as opposed to citronellol. G10H has also been documented to catalyze an irreversible oxidation of 10hydroxygeraniol [90], which might further contribute to its success in overcoming the geraniol bottleneck.

In addition to unwanted reductase activity from the IS, endogenous $S$. cerevisiae enzymes such as the reductase OYE2 and acetyltransferase Aft1 are known to degrade geraniol [57]. Of the two, OYE2 is the most active with its deletion resulting in $60 \%$ less geraniol degradation in yeast compared to Aft 1 with its deletion resulting in only a $7 \%$ reduction. Brown et al. saw a six-fold increase in strictosidine when they deleted these two genes [66]. By contrast, we saw no citronellol produced in our 10-hydroxygeraniol-producing strain expressing 10HGO (Figure 12A) indicating that OYE2 and OYE3 were not very active in our strain or that they were outcompeted by the CrG10H-Cr10HGO enzyme pair. Furthermore, our enzyme assay data using yeast crude lysates indicated that IS was $\sim 10$ times more potent at reducing geraniol than OYE2 and OYE3 combined and is probably the biggest drain on the pathway in our strain.

\subsection{Enzyme Co-localization and the MIA Pathway}

The unwanted activity of IS on geraniol and 10-hydroxygeraniol is a major barrier to reconstituting this pathway in yeast. There are many possible ways to solve this problem including protein engineering, promoter swapping as well as synthetic enzyme colocalization methods. We chose to investigate protein scaffolds as a solution, which can physically sequester enzymes together to improve turnover and reduce vulnerability to IS 
[91]. This strategy has been successfully used in other works, and has been documented to improve product yields up to 77 fold [67]. We begun by characterizing a set of 5 protein-protein binding domains selected from existing literature using a split Venus protein complementation assay. The results indicated that all domains successfully bound their ligands, with GBD, SYNZIP1/2 and SYNZIP3/4 being the most effective. We then showed we could redirect flux in the MIA pathway by scaffolding a section of the pathway spanning from GPP to citronellol. The base scaffold had an architecture consisting of SYN3-(GBD) $)_{x}-\mathrm{SH} 3$ and the number of GBD domains used varied from 1-3. Altering the number of GBD domains changes the enzymatic stoichiometry of the scaffold, with more GBD domains recruiting more copies of GES and increasing GPP turnover. Scaffold effectiveness did indeed prove to be proportional to the number of GBD domains used, with (GBD) 3 producing $117 \%$ more citronellol than (GBD) $)_{1}$ and $57.8 \%$ more citronellol relative to the unscaffolded control. So far only one other scaffolding experiment has been done in yeast, where the authors targeted resveratrol biosynthesis, and managed to improve the yield of that product five fold [68]. While our improvements were more modest, the efficiency of the scaffold is expected to be highly case-specific and is dependent on the architectural configuration of the entire macroprotein complex. In this case, the reduced efficiency of the SYN3-(GBD) ${ }_{1}-\mathrm{SH} 3$ scaffold relative to the control suggests that association with this scaffold may reduce the processivity of some or all of these enzymes. Despite this, the split-Venus interaction assay and successful functional tests on citronellol indicate that protein scaffolds are a useful tool for directing metabolic flux in Saccharomyces cerevisiae. Future work will focus on targeting the section of the pathway spanning from geraniol to iridodial, which are sensitive to IS promiscuity.

\subsection{Outlook}

MIAs of medicinal value have very complex structures that require long biosynthesis pathways. For example, strictosidine undergoes 6 to 8 proposed enzymatic steps to form 
catharanthine and/or tabersonine, while 7 enzymatic steps [64] convert tabersonine to vindoline and a peroxidase may be involved in the assembly of $\square-3^{\prime}, 4^{\prime}$ anhydrovinblastine from catharanthine and vindoline [92]. Recently, a prototype 7 step pathway was assembled in yeast to that converted tabersonine to several pathway intermediates and to vindoline. The results presented in this and in other studies $[66,86]$ suggest that a very efficient strictosidine pathway together with more efficient downstream steps [64] will be necessary in order to reach meaningful titers of different MIAs in yeast. In this work we assembled a pathway for the synthesis of the Iridiod nepetalactol in yeast and dissected its carbon distribution thereby identifying promiscuous reactions from $\mathrm{G} 10 \mathrm{H}$ and IS that severely impede flux through the pathway. As a first step towards solving this problem, we also demonstrated that protein scaffolds could be used to direct metabolic flux within the MIA pathway. We showed that scaffolds could be used to improve citronellol by $57.8 \%$, which serves as an excellent proof of concept towards their use in improving the function of IS sensitive enzymes. This information will be vital for further work on the reconstruction and optimization of MIA biosynthesis pathways in yeast.

\section{REFERENCES}

[1] P. J. Facchini and V. De Luca, "Opium poppy and Madagascar periwinkle: model non-model systems to investigate alkaloid biosynthesis in plants," Plant J, vol. $54,2008$.

[2] X.-J. Zhou and R. Rahmani, "Preclinical and Clinical Pharmacology of Vinca Alkaloids," Drugs, vol. 44, no. 4, pp. 1-16, 1992.

[3] P. J. Loehrer, S. D. Williams, and L. H. Einhorn, "Testicular Cancer: The Quest Continues," J. Natl. Cancer Inst., vol. 80, no. 17, pp. 1373-1382, Nov. 1988.

[4] V. T. DeVita and S. M. Hubbard, "Hodgkin's Disease," N. Engl. J. Med., vol. 328, no. 8, pp. 560-565, Feb. 1993.

[5] M. H. Veeder, J. R. Jett, J. Q. Su, J. A. Mailliard, J. F. Foley, R. J. Dalton, P. S. Etzell, R. F. Marschke, C. G. Kardinal, A. W. Maksymiuk, L. P. Ebbert, H. D. Tazelaar, and G. A. Witrak, "A phase III trial of mitomycin $\mathrm{C}$ alone versus mitomycin $\mathrm{C}$, vinblastine, and cisplatin for metastatic squamous cell lung carcinoma," Cancer, vol. 70, no. 9, pp. 2281-2287, 1992. 
[6] World Health Organization, "WHO Model List of Essential Medicines." Apr2015.

[7] T. Efferth, P. C. H. Li, V. S. B. Konkimalla, and B. Kaina, "From traditional Chinese medicine to rational cancer therapy," Trends Mol. Med., vol. 13, no. 8, pp. 353361, Aug. 2007.

[8] E. Elisabetsky and L. Costa-Campos, "The Alkaloid Alstonine: A Review of Its Pharmacological Properties," Evid. Based Complement. Alternat. Med., vol. 3, no. 1, pp. 39-48, Mar. 2006.

[9] A. Lorence and C. L. Nessler, "Camptothecin, over four decades of surprising findings," Phytochemistry, vol. 65, no. 20, pp. 2735-2749, Oct. 2004.

[10] M. Stiborová, C. A. Bieler, M. Wiessler, and E. Frei, "The anticancer agent ellipticine on activation by cytochrome P450 forms covalent DNA adducts $\downarrow 1$,", Biochem. Pharmacol., vol. 62, no. 12, pp. 1675-1684, Dec. 2001.

[11] E. S Akinboye and O. Bakare, "Biological activities of emetine," Open Nat. Prod. J., vol. 4, no. 1, 2011.

[12] S. E. Coplen, E. M. Antman, J. A. Berlin, P. Hewitt, and T. C. Chalmers, "Efficacy and safety of quinidine therapy for maintenance of sinus rhythm after cardioversion. A meta-analysis of randomized control trials.," Circulation, vol. 82, no. 4, pp. 1106-1116, 1990.

[13] J. Achan, A. O. Talisuna, A. Erhart, A. Yeka, J. K. Tibenderana, F. N. Baliraine, P. J. Rosenthal, and U. D'Alessandro, "Quinine, an old anti-malarial drug in a modern world: role in the treatment of malaria," Malar. J., vol. 10, no. 1, p. 1, 2011.

[14] R. Fife, J. C. Maclaurin, and J. H. Wright, "Rescinnamine in Treatment of Hypertension in Hospital Clinic and in General Practice," Br. Med. J., vol. 2, no. 5216, pp. 1848-1850, Dec. 1960.

[15] M. Bourin, M. Poncelet, R. Chermat, and P. Simon, "The value of the reserpine test in psychopharmacology.," Arzneimittelforschung., vol. 33, no. 8, pp. 1173$1176,1982$.

[16] W. Cole, "An introduction to diallyl-nor-toxiferene, a blocking muscle relaxant," Med. J. Aust., vol. 2, no. 6, p. 268-270, Aug. 1966.

[17] A. M. Caravaggi, A. Sardi, E. Baldoli, G. F. Di Francesco, and T. C. Luca, "Hemodynamic profile of a new cerebral vasodilator, vincamine and of one of its derivatives, apovincaminic acid ethylester (RGH-4405).," Arch. Int. Pharmacodyn. Ther., vol. 226, no. 1, pp. 139-148, 1977.

[18] S. W. Tam, M. Worcel, and M. Wyllie, "Yohimbine: a clinical review," Pharmacol. Ther., vol. 91, no. 3, pp. 215-243, 2001.

[19] Y. H. Choi, K.-P. Yoo, and J. Kim, "Supercritical Fluid Extraction and Liquid Chromatography-Electrospray Mass Analysis of Vinblastine from Catharanthus roseus," Chem. Pharm. Bull. (Tokyo), vol. 50, no. 9, pp. 1294-1296, 2002.

[20] F. Mu, L. Yang, W. Wang, M. Luo, Y. Fu, X. Guo, and Y. Zu, "Negative-pressure cavitation extraction of four main vinca alkaloids from Catharanthus roseus leaves," Molecules, vol. 17, no. 8, pp. 8742-8752, 2012.

[21] L. Yang, H. Wang, Y. Zu, C. Zhao, L. Zhang, X. Chen, and Z. Zhang, "Ultrasoundassisted extraction of the three terpenoid indole alkaloids vindoline, 
catharanthine and vinblastine from Catharanthus roseus using ionic liquid aqueous solutions," Chem. Eng. J., vol. 172, no. 2-3, pp. 705-712, Aug. 2011.

[22] K. Miettinen, L. Dong, N. Navrot, T. Schneider, V. Burlat, J. Pollier, L. Woittiez, S. van der Krol, R. Lugan, T. Ilc, R. Verpoorte, K.-M. Oksman-Caldentey, E.

Martinoia, H. Bouwmeester, A. Goossens, J. Memelink, and D. Werck-Reichhart, "The seco-iridoid pathway from Catharanthus roseus.," Nat. Commun., vol. 5, p. 3606, Jan. 2014.

[23] R. van Der Heijden, D. I. Jacobs, W. Snoeijer, D. Hallard, and R. Verpoorte, “The Catharanthus alkaloids: pharmacognosy and biotechnology," Curr Med Chem, vol. 11, 2004.

[24] M. S. for H. MSH, "International Drug Price Indicator Guide, Vinblastine Sulfate," 2014.

[25] H. Ishikawa, D. A. Colby, S. Seto, P. Va, A. Tam, H. Kakei, T. J. Rayl, I. Hwang, and D. L. Boger, "Total Synthesis of Vinblastine, Vincristine, Related Natural Products , and Key Structural Analogues," no. 4, pp. 4904-4916, 2009.

[26] T. Miyazaki, S. Yokoshima, S. Simizu, H. Osada, H. Tokuyama, and T. Fukuyama, "Synthesis of (+)-Vinblastine and Its Analogues," Org. Lett., vol. 9, no. 23, pp. 4737-4740, Nov. 2007.

[27] I.-M. Chung, E.-H. Kim, M. Li, C. a M. Peebles, W.-S. Jung, H.-K. Song, J.-K. Ahn, and K.-Y.San, "Screening 64 cultivars Catharanthus roseus for the production of vindoline, catharanthine, and serpentine.," Biotechnol. Prog., vol. 27, no. 4, pp. 937-43, Jul. 2011.

[28] T. Srivastava, S. Das, S. K. Sopory, and P. S. Srivastava, "A reliable protocol for transformation of Catharanthus roseus through Agrobacterium tumefaciens," Physiol Mol Biol Plants, vol. 15, 2009.

[29] G. Guirimand, V. Burlat, A. Oudin, A. Lanoue, B. St-Pierre, and V. Courdavault, "Optimization of the transient transformation of Catharanthus roseus cells by particle bombardment and its application to the subcellular localization of hydroxymethylbutenyl 4-diphosphate synthase and geraniol 10-hydroxylase," Plant Cell Rep, vol. 28, 2009.

[30] F. Hilliou, P. Christou, and M. J. Leech, "Development of an efficient transformation system for Catharanthus roseus cell cultures using particle bombardment," Plant Sci, vol. 140, 1999.

[31] Q. Pan, Q. Wang, F. Yuan, S. Xing, J. Zhao, Y. H. Choi, R. Verpoorte, Y. Tian, G. Wang, and K. Tang, "Overexpression of ORCA3 and G10H in Catharanthus roseus plants regulated alkaloid biosynthesis and metabolism revealed by NMR-metabolomics.," PloS One, vol. 7, no. 8, p. e43038, Jan. 2012.

[32] R. Zárate and R. Verpoorte, "Strategies for the genetic modification of the medicinal plant Catharanthus roseus (L.) G. Don," Phytochem. Rev., vol. 6, no. 23, pp. 475-491, Mar. 2007.

[33] S. Hellwig, J. Drossard, R. M. Twyman, and R. Fischer, "Plant cell cultures for the production of recombinant proteins.," Nat. Biotechnol., vol. 22, no. 11, pp. 1415-22, Nov. 2004. 
[34] R. Verpoorte, A. Contin, and J. Memelink, "Biotechnology for the production of plant secondary metabolites," Phytochem. Rev., vol. 1, no. 1, pp. 13-25, 2002.

[35] L. Almagro, A. B. Sabater-Jara, S. Belchí-Navarro, F. Fernández-Pérez, R. Bru, and M. A. Pedreño, "Effect of UV light on secondary metabolite biosynthesis in plant cell cultures elicited with cyclodextrins and methyljasmonate," Plants Environ. Intech, pp. 115-136, 2011.

[36] S. Mahroug, V. Burlat, and B. St-Pierre, "Cellular and sub-cellular organisation of the monoterpenoid indole alkaloid pathway in Catharanthus roseus," Phytochem. Rev., vol. 6, no. 2, pp. 363-381, 2007.

[37] P. J. Westfall, D. J. Pitera, J. R. Lenihan, D. Eng, F. X. Woolard, R. Regentin, T. Horning, H. Tsuruta, D. J. Melis, A. Owens, S. Fickes, D. Diola, K. R. Benjamin, J. D. Keasling, M. D. Leavell, D. J. McPhee, N. S. Renninger, J. D. Newman, and C. J. Paddon, "Production of amorphadiene in yeast, and its conversion to dihydroartemisinic acid, precursor to the antimalarial agent artemisinin.," Proc. Natl. Acad. Sci. U. S. A., vol. 109, no. 3, pp. E111-8, Jan. 2012.

[38] W. C. DeLoache, Z. N. Russ, L. Narcross, A. M. Gonzales, V. J. J. Martin, and J. E. Dueber, "An enzyme-coupled biosensor enables (S)-reticuline production in yeast from glucose," Nat. Chem. Biol., vol. 11, no. 7, pp. 465-471, May 2015.

[39] E. Fossati, L. Narcross, A. Ekins, J.-P. Falgueyret, and V. J. J. Martin, "Synthesis of Morphinan Alkaloids in Saccharomyces cerevisiae.," PloS One, vol. 10, no. 4, p. e0124459, Jan. 2015.

[40] S. Galanie, K. Thodey, I. J. Trenchard, M. Filsinger Interrante, A. Choad, and S. Smolke, "Complete biosynthesis of opioids in yeast," Science, vol. 349, no. 6252, pp. 1095-1100, 2015.

[41] K. Asada, V. Salim, S. Masada-Atsumi, E. Edmunds, M. Nagatoshi, K. Terasaka, H. Mizukami, and V. De Luca, "A 7-Deoxyloganetic Acid Glucosyltransferase Contributes a Key Step in Secologanin Biosynthesis in Madagascar Periwinkle," Plant Cell, vol. 25, no. 10, pp. 4123-4134, Oct. 2013.

[42] V. Salim, B. Wiens, S. Masada-Atsumi, F. Yu, and V. D. Luca, "7-Deoxyloganetic acid synthase catalyzes a key 3 step oxidation to form 7-deoxyloganetic acid in Catharanthus roseus iridoid biosynthesis," Phytochemistry, vol. 101, pp. 23 31, 2014.

[43] V. Salim, F. Yu, J. Altarejos, and V. Luca, "Virus - induced gene silencing identifies Catharanthus roseus 7 - deoxyloganic acid - 7 - hydroxylase, a step in iridoid and monoterpene indole alkaloid biosynthesis," Plant J., vol. 76, no. 5, pp. 754-765, 2013.

[44] G. H. Braus, "Aromatic amino acid biosynthesis in the yeast Saccharomyces cerevisiae: a model system for the regulation of a eukaryotic biosynthetic pathway.," Microbiol. Rev., vol. 55, no. 3, pp. 349-370, Sep. 1991.

[45] W. Noé, C. Mollenschott, and J. Berlin, "Tryptophan decarboxylase from Catharanthus roseus cell suspension cultures: purification, molecular and kinetic data of the homogenous protein," Plant Mol. Biol., vol. 3, no. 5, pp. 281288, 1984. 
[46] I. Buhaescu and H. Izzedine, "Mevalonate pathway: A review of clinical and therapeutical implications," Clin. Biochem., vol. 40, no. 9-10, pp. 575-584, Jun. 2007.

[47] L. Kizer, D. J. Pitera, B. F. Pfleger, and J. D. Keasling, "Application of functional genomics to pathway optimization for increased isoprenoid production.," Appl. Environ. Microbiol., vol. 74, no. 10, pp. 3229-41, May 2008.

[48] Y. a Primak, M. Du, M. C. Miller, D. H. Wells, A. T. Nielsen, W. Weyler, and Z. Q. Beck, "Characterization of a feedback-resistant mevalonate kinase from the archaeon Methanosarcina mazei.," Appl. Environ. Microbiol., vol. 77, no. 21, pp. 7772-8, Nov. 2011.

[49] O. Herrero, D. Ramón, and M. Orejas, "Engineering the Saccharomyces cerevisiae isoprenoid pathway for de novo production of aromatic monoterpenes in wine.," Metab. Eng., vol. 10, no. 2, pp. 78-86, Mar. 2008.

[50] L. Berkeley, "Lawrence Berkeley National Laboratory Peer Reviewed Title : Biofuel alternatives to ethanol : pumping the microbial well Author : Publication Date : Permalink : Preferred Citation : Local Identifier : LBNL Paper LBNL-3256E Abstract: Engineered microorgan," 2010.

[51] V. J. J. Martin, D. J. Pitera, S. T. Withers, J. D. Newman, and J. D. Keasling, "Engineering a mevalonate pathway in Escherichia coli for production of terpenoids," Nat Biotech, vol. 21, no. 7, pp. 796-802, Jul. 2003.

[52] C. Chambon, V. Ladeveze, A. Oulmouden, M. Servouse, and E. Karst, "Isolation and properties of yeast mutants affected in farnesyl diphosphate synthetase," Curr. Genet., vol. 100, no. 18, pp. 41-46, 1990.

[53] C. Burke and R. Croteau, "Geranyl diphosphate synthase from Abies grandis: cDNA isolation, functional expression, and characterization," Arch Biochem Biophys, vol. 405, no. 1, pp. 130-136, 2002.

[54] K. Grabin, M. Kwapisz, J. Sikora, M. Boguta, W. J. Smagowicz, and Z. Teresa, "The isoprenoid biosynthetic pathway in Saccharomyces cerevisiae is a affected in a maf1-1 mutant with altered tRNA synthesis," FEMS Yeast Res., vol. 2, pp. 31-37, 2002.

[55] S. Takahashi, Y. Yeo, B. T. Greenhagen, T. McMullin, L. Song, J. Maurina-Brunker, R. Rosson, J. P. Noel, and J. Chappell, "Metabolic Engineering of Sesquiterpene Metabolism in Yeast," Biotechnol. Bioeng., vol. 97, no. 1, pp. 170-181, May 2007.

[56] P. Amiri, A. Shahpiri, M. A. Asadollahi, F. Momenbeik, and S. Partow, "Metabolic engineering of Saccharomyces cerevisiae for linalool production," Biotechnol. Lett., vol. 38, no. 3, pp. 503-508, 2016.

[57] D. Steyer, C. Erny, P. Claudel, G. Riveill, F. Karst, and J.-L. Legras, "Genetic analysis of geraniol metabolism during fermentation," Food Microbiol., vol. 33, no. 2, pp. 228-234, Apr. 2013.

[58] E. Jongedijk, K. Cankar, J. Ranzin, and S. Van der Krol, "Capturing of the monoterpene olefin limonene produced in Saccharomyces cerevisiae."

[59] G. Collu, N. Unver, A. M. G. Peltenburg-Looman, R. van der Heijden, R. Verpoorte, and J. Memelink, "Geraniol 10-hydroxylase1, a cytochrome P450 
enzyme involved in terpenoid indole alkaloid biosynthesis," FEBS Lett., vol. 508, no. 2, pp. 215-220, 2001.

[60] R. Krithika, P. L. Srivastava, B. Rani, S. P. Kolet, M. Chopade, M. Soniya, and H. V. Thulasiram, "Characterization of 10-Hydroxygeraniol Dehydrogenase from Catharanthus roseus Reveals Cascaded Enzymatic Activity in Iridoid Biosynthesis," Sci. Rep., vol. 5, p. 8258, Feb. 2015.

[61] A. Geerlings, M. M.-L. Ibañez, J. Memelink, R. van der Heijden, and R. Verpoorte, "Molecular Cloning and Analysis of Strictosidine $\beta$-d-Glucosidase, an Enzyme in Terpenoid Indole Alkaloid Biosynthesis in Catharanthus roseus," J. Biol. Chem., vol. 275, no. 5, pp. 3051-3056, Feb. 2000.

[62] R. T. Brown, J. S. Hill, G. F. Smith, and K. S. J. Stapleford, "On the rearrangement of catharanthine, stemmadenine and tabersonine in acetic acid," Tetrahedron, vol. 27, no. 21, pp. 5217-5228, Jan. 1971.

[63] M. El-Sayed, Y. H. Choi, M. Frédérich, S. Roytrakul, and R. Verpoorte, "Alkaloid accumulation in Catharanthus roseus cell suspension cultures fed with stemmadenine," Biotechnol. Lett., vol. 26, no. 10, pp. 793-798, 2004.

[64] Y. Qu, M. L. A. E. Easson, J. Froese, R. Simionescu, T. Hudlicky, and V. De Luca, "Completion of the seven-step pathway from tabersonine to the anticancer drug precursor vindoline and its assembly in yeast," Proc. Natl. Acad. Sci. U. S. A., vol. 112, no. 19, pp. 6224-6229, May 2015.

[65] M. Sottomayor, M. López-Serrano, F. DiCosmo, and A. Ros Barceló, "Purification and characterization of $\alpha-3^{\prime}, 4^{\prime}$-anhydrovinblastine synthase (peroxidaselike) from Catharanthus roseus (L.) G. Don," FEBS Lett., vol. 428, no. 3, pp. 299303, May 1998.

[66] S. Brown, M. Clastre, V. Courdavault, and S. E. O'Connor, "De novo production of the plant-derived alkaloid strictosidine in yeast.," Proc. Natl. Acad. Sci. U. S. A., no. 27, pp. 1-6, Feb. 2015.

[67] J. E. Dueber, G. C. Wu, G. R. Malmirchegini, T. S. Moon, C. J. Petzold, A. V. Ullal, K. L. J. Prather, and J. D. Keasling, "Synthetic protein scaffolds provide modular control over metabolic flux.," Nat. Biotechnol., vol. 27, no. 8, pp. 753-9, Aug. 2009.

[68] Y. Wang and 0. Yu, "Synthetic scaffolds increased resveratrol biosynthesis in engineered yeast cells.," J. Biotechnol., vol. 157, no. 1, pp. 258-60, Jan. 2012.

[69] J. T. Nguyen, C. W. Turck, F. E. Cohen, R. N. Zuckermann, and W. a Lim, "Exploiting the basis of proline recognition by SH3 and WW domains: design of N-substituted inhibitors.," Science, vol. 282, no. 5396, pp. 2088-92, Dec. 1998.

[70] N. Abdul-Manan, B. Aghazadeh, G. a Liu, a Majumdar, O. Ouerfelli, K. a Siminovitch, and M. K. Rosen, "Structure of Cdc42 in complex with the GTPasebinding domain of the 'Wiskott-Aldrich syndrome' protein.," Nature, vol. 399, no. 6734, pp. 379-83, May 1999.

[71] K. E. Thompson, C. J. Bashor, W. a Lim, and A. E. Keating, "SYNZIP Protein Interaction Toolbox: in Vitro and in Vivo Specifications of Heterospecific 
Coiled-Coil Interaction Domains.," ACS Synth. Biol., vol. 1, no. 4, pp. 118-129, Apr. 2012.

[72] W. P. Russ, D. M. Lowery, P. Mishra, M. B. Yaffe, and R. Ranganathan, "Naturallike function in artificial WW domains.," Nature, vol. 437, no. 7058, pp. 579-83, Sep. 2005.

[73] M. P. DeLisa and R. Conrado, "Synthetic metabolic pipelines," Nat. Biotechnol., vol. 27, pp. 728-729, 2009.

[74] Z. Shao, H. Zhao, and H. Zhao, "DNA assembler, an in vivo genetic method for rapid construction of biochemical pathways," Nucleic Acids Res., vol. 37, no. 2, pp. e16-e16, Feb. 2009.

[75] R. D. Gietz and R. H. Schiestl, "High-efficiency yeast transformation using the LiAc/SS carrier DNA/PEG method.," Nat. Protoc., vol. 2, no. 1, pp. 31-4, Jan. 2007.

[76] Z. H. Shao Z, "DNA assembler, an in vivo genetic method for rapid construction of biochemical pathways.," Nucleic Acids Res, vol. 37:e16, 2009.

[77] X. Li, Z. Cheng, and H. Jin, "Dynamics of Ras Complexes Observed in Living Cells," Sensors, vol. 12, no. 1, pp. 9411-9422, 2012.

[78] M. J. C. Fischer, S. Meyer, P. Claudel, M. Perrin, J. F. Ginglinger, C. Gertz, J. E. Masson, D. Werck-Reinhardt, P. Hugueney, and F. Karst, "Specificity of Ocimum basilicum geraniol synthase modified by its expression in different heterologous systems.," J. Biotechnol., vol. 163, no. 1, pp. 24-9, Jan. 2013.

[79] D. D. Hinson, K. L. Chambliss, M. J. Toth, R. D. Tanaka, and K. M. Gibson, "Posttranslational regulation of mevalonate kinase by intermediates of the cholesterol and nonsterol isoprene biosynthetic pathways.," J. Lipid Res., vol. 38, no. 11, pp. 2216-2223, Nov. 1997.

[80] M. J. C. Fischer, S. Meyer, P. Claudel, M. Bergdoll, and F. Karst, "Metabolic engineering of monoterpene synthesis in yeast," Biotechnol. Bioeng., vol. 108, no. 8, pp. 1883-1892, 2011.

[81] F. D. O. Pereira, J. M. Mendes, I. O. Lima, K. S. D. L. Mota, W. A. D. Oliveira, and E. D. O. Lima, "Antifungal activity of geraniol and citronellol, two monoterpenes alcohols, against Trichophyton rubrum involves inhibition of ergosterol biosynthesis.," Pharm. Biol., vol. 53, no. 2, pp. 228-34, Feb. 2015.

[82] R. Nes, C. Sekula, W. D. Nes, and J. H. Adler, "The Functional Yeast* Importance of Structural Features of Ergosterol in," 1978.

[83] R. Höfer, B. Boachon, H. Renault, C. Gavira, L. Miesch, J. Iglesias, J.-F. Ginglinger, L. Allouche, M. Miesch, S. Grec, R. Larbat, and D. Werck-Reichhart, "Dual function of the cytochrome P450 CYP76 family from Arabidopsis thaliana in the metabolism of monoterpenols and phenylurea herbicides.," Plant Physiol., vol. 166, no. 3, pp. 1149-61, Dec. 2014.

[84] N. D. Gold, C. M. Gowen, F.-X. Lussier, S. C. Cautha, R. Mahadevan, and V. J. J. Martin, "Metabolic engineering of a tyrosine-overproducing yeast platform using targeted metabolomics.," Microb. Cell Factories, vol. 14, p. 73, Jan. 2015.

[85] M. A. H. Luttik, Z. Vuralhan, E. Suir, G. H. Braus, J. T. Pronk, and J. M. Daran, "Alleviation of feedback inhibition in Saccharomyces cerevisiae aromatic amino 
acid biosynthesis: Quantification of metabolic impact," Metab. Eng., vol. 10, no. 3-4, pp. 141-153, May 2008.

[86] F. Geu-Flores, N. H. Sherden, V. Courdavault, V. Burlat, W. S. Glenn, C. Wu, E. Nims, Y. Cui, and S. E. O'Connor, "An alternative route to cyclic terpenes by reductive cyclization in iridoid biosynthesis.," Nature, vol. 492, no. 7427, pp. 138-42, Dec. 2012.

[87] E. W. Trotter, E. J. Collinson, I. W. Dawes, and C. M. Grant, "Old yellow enzymes protect against acrolein toxicity in the yeast Saccharomyces cerevisiae.," Appl. Environ. Microbiol., vol. 72, no. 7, pp. 4885-92, Jul. 2006.

[88] T. S. Moon, J. E. Dueber, E. Shiue, and K. L. J. Prather, "Use of modular, synthetic scaffolds for improved production of glucaric acid in engineered E. coli.," Metab. Eng., vol. 12, no. 3, pp. 298-305, May 2010.

[89] M. Oswald, M. Fischer, N. Dirninger, and F. Karst, "Monoterpenoid biosynthesis in Saccharomyces cerevisiae.," FEMS Yeast Res., vol. 7, no. 3, pp. 413-21, May 2007.

[90] R. Höfer, L. Dong, F. André, J. Ginglinger, R. Lugan, C. Gavira, S. Grec, G. Lang, J. Memelink, S. V. D. Krol, H. Bouwmeester, and D. Werck-reichhart, "Geraniol hydroxylase and hydroxygeraniol oxidase activities of the CYP76 family of cytochrome P450 enzymes and potential for engineering the early steps of the ( seco ) iridoid pathway," Metab. Eng., vol. 20, pp. 221-232, 2013.

[91] L. Narcross, E. Fossati, L. Bourgeois, J. E. Dueber, and V. J. J. Martin, "Microbial Factories for the Production of Benzylisoquinoline Alkaloids.," Trends Biotechnol., vol. xx, pp. 1-14, Jan. 2016.

[92] M. M. R. Costa, F. Hilliou, P. Duarte, L. G. Pereira, I. Almeida, M. Leech, J. Memelink, A. R. Barceló, and M. Sottomayor, "Molecular Cloning and Characterization of a Vacuolar Class III Peroxidase Involved in the Metabolism of Anticancer Alkaloids in Catharanthus roseus," Plant Physiol., vol. 146, no. 2, pp. 403-417, Feb. 2008. 